\title{
Perfluorooctanoic acid (PFOA), perfluorooctane sulfonic acid (PFOS), and perfluorononanoic acid (PFNA) increase triglyceride levels and decrease cholesterogenic gene expression in human HepaRG liver cells
}

\author{
Jochem Louisse ${ }^{1}$ (1) $\cdot$ Deborah Rijkers $^{1} \cdot$ Geert Stoopen ${ }^{1} \cdot$ Aafke Janssen $^{1} \cdot$ Martijn Staats $^{1} \cdot$ Ron Hoogenboom $^{1}$. \\ Sander Kersten ${ }^{2} \cdot$ Ad Peijnenburg $^{1}$
}

Received: 13 April 2020 / Accepted: 15 June 2020 / Published online: 25 June 2020

(c) The Author(s) 2020

\begin{abstract}
Per- and polyfluoroalkyl substances (PFASs) are omnipresent in the environment, food chain, and humans. Epidemiological studies have shown a positive association between serum levels of perfluorooctanoic acid (PFOA) and perfluorooctane sulfonic acid (PFOS), and increased serum cholesterol and, in some cases, also triglyceride levels. However, causality has been questioned, as animal studies, as well as a human trial, showed a decrease in serum cholesterol and no effects or a decrease in plasma triglycerides. To obtain more insight into the effects of PFASs on these processes, the present study investigated the effects of PFOA, PFOS, and perfluorononanoic acid (PFNA) on intracellular triglyceride and cholesterol levels in human HepaRG liver cells. DNA microarray analyses were performed to provide insight into underlying mechanisms. All PFASs induced an increase in cellular triglyceride levels, but had no effect on cholesterol levels. Gene set enrichment analysis (GSEA) of the microarray data indicated that gene sets related to cholesterol biosynthesis were repressed by PFOA, PFOS, and PFNA. Other gene sets commonly affected by all PFAS were related to PERK/ATF4 signaling (induced), tRNA aminoacylation (induced), amino acid transport (induced), and glycolysis/gluconeogenesis (repressed). Moreover, numerous target genes of peroxisome proliferator-activated receptor $\alpha(\operatorname{PPAR} \alpha)$ were found to be upregulated. Altogether, the present study shows that PFOA, PFOS, and PFNA increase triglyceride levels and inhibit cholesterogenic gene expression in HepaRG cells. In addition, the present study indicates that PFASs induce endoplasmic reticulum stress, which may be an important mechanism underlying some of the toxic effects of these chemicals.
\end{abstract}

Keywords PFASs $\cdot$ HepaRG cells $\cdot$ Transcriptomics $\cdot$ Cholesterol $\cdot$ Triglycerides

\section{Introduction}

Per- and polyfluoroalkyl substances (PFASs) are man-made chemicals that are extremely persistent and omnipresent in the environment (Wang et al. 2017). PFASs contain at least one fluoro-carbon chain of different lengths with varying

Electronic supplementary material The online version of this article (https://doi.org/10.1007/s00204-020-02808-0) contains supplementary material, which is available to authorized users.

Jochem Louisse

jochem.louisse@wur.nl

1 Wageningen Food Safety Research (WFSR), Wageningen, The Netherlands

2 Nutrition, Metabolism and Genomics Group, Division of Human Nutrition and Health, Wageningen University, Wageningen, The Netherlands chemical groups attached, and have unique chemical and physical characteristics, such as oil and water repellency, high temperature and chemical resistance, and emulsifying/ surfactant properties. Because of these favorable properties, they are widely used in various industrial and consumer applications, e.g., firefighting foams, electronics, textiles, food contact materials, and cosmetics. According to OECD (2018), over 4700 PFASs have been identified. The production and use of the best-known and most-studied PFASs, 
perfluorooctanoic acid (PFOA) and perfluorooctane sulfonate (PFOS), have been restricted globally due to concerns of risks to human health and the environment (ATSDR 2018; EFSA CONTAM Panel 2018). However, even though the use of PFOS and PFOA has been restricted, recent assessments of the European Food Safety Authority (EFSA CONTAM Panel 2018, 2020) state that a considerable proportion of the European population is exposed to higher PFAS levels than the tolerably weekly intake (TWI) values.

EFSA CONTAM Panel (2018) derived TWIs for PFOS and PFOA of, respectively, 13 and $6 \mathrm{ng} / \mathrm{kw}$ bw/week, based on the positive association between serum PFAS levels and serum cholesterol. This was observed in numerous epidemiological studies, including those by Steenland et al. (2009), Nelson et al. (2010), and Eriksen et al. (2013), studies that were used to derive $\mathrm{BMDL}_{5}$ serum levels of $21-25 \mathrm{ng} / \mathrm{mL}$ for PFOS and 9.2-9.4 $\mathrm{ng} / \mathrm{mL}$ for PFOA. Corresponding daily intakes resulting in these serum levels at adult age were established using PBPK modeling, which were then used to derive the two TWIs. An increase in cholesterol levels was seen as adverse, since it is regarded to be a risk factor for cardiovascular disease. Besides an increase in serum cholesterol, exposure to PFOS and PFOA is also positively associated with an increase in serum triglyceride levels in a number of epidemiological studies (EFSA CONTAM Panel 2018). Interestingly, various rat studies, including those by NTP $(2019 a, b)$, have shown that exposure to PFASs decreases rather than increases serum cholesterol and triglyceride levels. A similar observation was made in monkeys treated with PFOS (Seacat et al. 2002). Furthermore, data of a recent study on the kinetics and effects of PFOA in human cancer patients in a phase 1 dose-escalation trial also indicate that PFOA reduces serum cholesterol levels, whereas serum triglyceride levels were unaffected (Convertino et al. 2018). These human data are in line with data from a study using APOE*3-Leiden.CETP mice, a model with a human-like lipoprotein metabolism, in which PFOA decreased plasma triglycerides, total cholesterol, and nonHDL-C, whereas HDL-C was increased (Pouwer et al. 2019). It should be noted that in these studies, the exposure and resulting serum levels are much higher than observed in epidemiological studies. Regarding the contradictory findings on the relation between PFAS exposure and cholesterol levels, Convertino et al. (2018) suggested several confounding factors that may explain the observed associations. After the publication of the EFSA Opinion (EFSA Contam Panel 2018), another potential confounding mechanism related to the enterohepatic cycling of both bile acids and PFASs was brought up (https://www.efsa.europa.eu/sites/default/files /news/efsa-contam-3503.pdf). More studies are needed to investigate the underlying mechanisms to support a causal relationship, as also recommended by EFSA in its recent risk assessment (EFSA CONTAM Panel 2020). It is noteworthy that in this new risk assessment, a group TWI of $8 \mathrm{ng} / \mathrm{kg}$ bw per week for the sum of four PFASs (PFOA, PFNA, PFHxS, and PFOS) was proposed, based on the effects on the immune system that were also associated with rather low serum levels of PFASs.

In light of the uncertainties around the relation between PFAS exposure and cholesterol and triglyceride levels, a better understanding of how PFASs may interfere with cholesterol and triglyceride metabolism is thus required. Inasmuch as the liver plays an important role in the regulation of cholesterol and triglyceride levels, mechanistic in vitro studies with human liver cells are important to gain insight into the effects of PFASs on these processes. In animals, PFASs have been shown to cause hypertrophy and hyperplasia of the liver, but also steatosis and possibly cholestasis (EFSA CONTAM Panel 2018, 2020). To obtain more insight into the effects of PFASs on the human liver, the effects of PFOS and PFOA and the related PFAS perfluorononanoic acid (PFNA) in human HepaRG liver cells were determined in the present study. Effects on PFASinduced changes in cellular cholesterol and triglyceride levels were assessed, followed by transcriptomics analysis to gain insight into possible underlying mechanisms.

\section{Materials and methods}

\section{Chemicals}

PFOA (purity 99\%) and PFNA (purity 99\%) were purchased from Sigma-Aldrich (Zwijndrecht, The Netherlands) and PFOS (purity 100\%) was obtained from Synquest laboratories (Alachua FL). All stock solutions (dilution series) of the compounds were prepared in $100 \%$ dimethyl sulfoxide (DMSO HybriMax, Sigma-Aldrich). Chemical structures of the three PFASs are shown in Fig. 1.
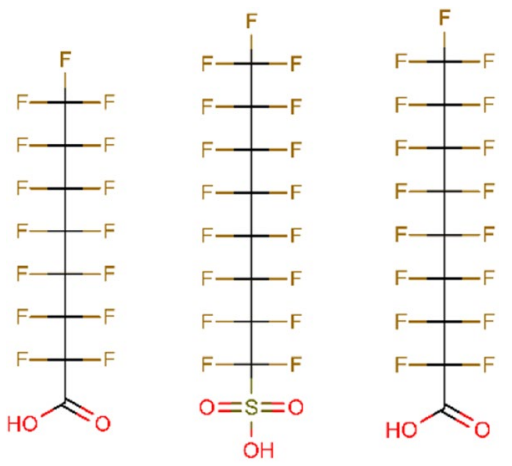

PFOA

PFOS

PFNA

Fig. 1 Chemical structures of the PFASs tested in the present study 


\section{HepaRG cell culture}

The human hepatic cell line HepaRG was obtained from Biopredic International (Rennes, France) and cultured in growth medium consisting of William's Medium E+GlutaMAX ${ }^{\text {TM }}$ (ThemoFisher Scientific, Landsmeer, The Netherlands) supplemented with $10 \%$ Good Forte filtrated bovine serum (FBS; PAN ${ }^{\mathrm{TM}}$ Biotech, Aidenbach, Germany), $1 \%$ PS (100 U/mL penicillin, $100 \mu \mathrm{g} / \mathrm{mL}$ streptomycin; Capricorn Scientific, Ebsdorfergrund, Germany), $50 \mu \mathrm{M}$ hydrocortisone hemisuccinate (sodium salt) (Sigma-Aldrich), and $5 \mu \mathrm{g} / \mathrm{mL}$ human insulin (PAN ${ }^{\mathrm{TM}}$ Biotech). Seeding, trypsinization [using $0.05 \%$ Trypsin-EDTA (ThermoFisher Scientific)], and maintenance of the cells was performed according to the HepaRG instruction manual from Biopredic International. For cell viability studies, HepaRG cells were seeded in black-coated 96-well plates (Greiner Bio-One, Frickenhausen, Germany; 9000 cells per well in $100 \mu \mathrm{L}$ ). For gene expression studies and analysis of triglyceride and cholesterol levels, HepaRG cells were seeded in 24-well plates (Corning, Corning, NY; 55,000 cells per well in $500 \mu \mathrm{L}$ ). After 2 weeks on growth medium, cells were cultured for two days in growth medium supplemented with $0.85 \%$ DMSO to induce differentiation. Subsequently, cells were cultured for 12 days in growth medium supplemented with $1.7 \%$ DMSO (differentiation medium) for final differentiation. At this stage, cells were ready to be used for toxicity studies. Cells that were not immediately used were kept on differentiation medium for a maximum of 3 additional weeks. Cell cultures were maintained in an incubator (humidified atmosphere with $5 \% \mathrm{CO}_{2}$ at $37^{\circ} \mathrm{C}$ ) and the medium was refreshed every $2-3$ days during culturing. Prior to toxicity studies, differentiated HepaRG cells were incubated for $24 \mathrm{~h}$ in assay medium (growth medium-containing 2\% FBS) supplemented with 0.5\% DMSO.

\section{Cell exposure}

Test chemicals were diluted from 200-fold concentrated stock solutions in the assay medium, providing a final DMSO concentration of $0.5 \%$. In each experiment, a solvent control $(0.5 \%$ DMSO) was included. PFASs were tested in concentrations up to $400 \mu \mathrm{M}$. Different exposure durations and concentrations were used. After exposure, the effects of the PFASs on cell viability, triglyceride, and cholesterol levels, and gene expression were assessed.

\section{Cell viability studies}

The effect of the chemicals on cell viability was in the first instance determined on PFAS-exposed HepaRG cells cultured in 96-well plates, using the WST-1 assay. This assay determines the conversion of the tetrazolium salt WST-1
(4-[3-(4-iodophenyl)-2-(4-nitrophenyl)-2H-5-tetrazolio]1,3-benzene disulfonate) to formazan by metabolically active cells. After exposure for 6,24 , or $72 \mathrm{~h}$, the medium was removed and the cells were washed with Dulbecco's Phosphate-Buffered Saline (DPBS; ThermoFisher Scientific). Next, WST-1 solution (Sigma-Aldrich) was added to the cell culture medium (1:10 dilution) and $100 \mu \mathrm{L}$ was added to each well. After $1 \mathrm{~h}$ incubation in an incubator (humidified atmosphere with $5 \% \mathrm{CO}_{2}$ at $37{ }^{\circ} \mathrm{C}$ ), the plate was shaken at $1000 \mathrm{rpm}$ for $1 \mathrm{~min}$, and absorbance at $450 \mathrm{~nm}$ was measured (background absorbance at $630 \mathrm{~nm}$ was subtracted) using a microplate reader (Synergy ${ }^{\mathrm{TM}}$ HT BioTek, Winooski, VT, USA). Further experiments (cholesterol and triglyceride analyses and gene expression analyses) were performed in 24-well plates. Therefore, possible effects on cell viability were also examined in HepaRG cells in 24-well plates, by determining the total DNA quantity in each well. To that end, cells were lysed with RLT-lysis buffer (Qiagen, Venlo, The Netherlands) and the Quant-iT ${ }^{\mathrm{TM}}$ PicoGreen ${ }^{\circledR}$ dsDNA Assay Kit (Life Technologies) was used for DNA quantification. The samples were diluted two times in DPBS. Samples were also diluted another two times (in 1:1 RLT:DPBS). TE reagent was prepared according to the manufacturer's protocol. A standard curve of dsDNA in TE reagent was prepared; a range of $1.25-0.002 \mu \mathrm{g} / \mathrm{mL}$ was used for quantification. In a black 96 -well plate, $5 \mu \mathrm{L}$ sample and $195 \mu \mathrm{L}$ TE reagent were added, protected from light. DPBS:RLT (1:1) was taken along as a blanc. The plate was mixed in the dark for $4 \mathrm{~min}$ at $1100 \mathrm{rpm}$, and afterwards incubated for $5 \mathrm{~min}$ at room temperature. Fluorescence was measured using a microplate reader (Synergy ${ }^{\mathrm{TM}}$ HT BioTek) at excitation $485 / 20 \mathrm{~nm}$, emission $528 / 20 \mathrm{~nm}$, and a sensitivity of 40 .

\section{Triglyceride and cholesterol analysis}

To determine the effect of PFAS exposure on triglyceride and cholesterol levels, a method based on gas chromatography with flame-ionization detection (GC-FID) was applied. To this end, differentiated HepaRG cells in 24-well culture plates were exposed for $24 \mathrm{~h}$ to PFOS, PFOA, or PFNA at concentrations ranging from 25 to $200 \mu \mathrm{M}$. After exposure, cells were washed three times with $0.5 \mathrm{~mL}$ DPBS/well. Cells were harvested in $150 \mu \mathrm{L}$ RLT-lysis buffer and lysates of two wells were pooled in $1.5 \mathrm{~mL}$ Eppendorf Protein LoBind microcentrifuge tubes (Fisher Scientific, Hampton, USA). The samples were stored at $-80{ }^{\circ} \mathrm{C}$ until extraction. The extraction of triglycerides was performed under an $\mathrm{N}_{2}$ atmosphere according to a method described by Hutchins et al. (2008) using isooctane:ethyl acetate (75:25). After thawing, the samples were transferred to $10 \mathrm{~mL}$ glass tubes. A mixture of isooctane and ethyl acetate $(75: 25,5 \mathrm{~mL})$ with $10 \mu \mathrm{L} 0.5 \mathrm{mg} / \mathrm{mL}$ tritridecanoin (Nu-Chek Prep Inc., Elysian, USA) was added to the samples, followed by vortex-mixing 
(30 s), sonication (60 s), vortex-mixing (30 s), and incubation head over head (15 min). $\mathrm{NaCl}(1 \mathrm{~mL}, 0.9 \% \mathrm{w} / \mathrm{v})$ was added and the samples were vortex-mixed for $60 \mathrm{~s}$ and centrifuged for $10 \mathrm{~min}$ at $1000 \times \mathrm{g}$ to separate the two phases. A total of $4.2 \mathrm{~mL}$ of the organic (upper) phase were collected with a 1-mL glass Hamilton syringe. The polar phase was extracted again by adding $2 \mathrm{~mL}$ isooctane:ethyl acetate (75:25) followed by vortex-mixing, centrifugation, and collection of the organic phase. The two organic phases were combined, dried under $\mathrm{N}_{2}$ gas at $30-37{ }^{\circ} \mathrm{C}$, redissolved in $100 \mu \mathrm{L}$ isooctane, and transferred to a $\mathrm{GC}$ vial. The samples were analyzed on a Trace GC Ultra GC-FID system (ThermoFisher Scientific, Waltham, USA). A sample volume of $2 \mu \mathrm{L}$ was injected on a $5 \mathrm{~m} \times 0.53 \mathrm{~mm}, 0.17 \mu \mathrm{m}$ Sim Dist Ulti Metal column (CP7532, Agilent Technologies, Santa Clara, USA) and eluted at a $\mathrm{N}_{2}$ gas flow of $45.5 \mathrm{~mL} / \mathrm{min}$. The temperature program was as follows: $0.5 \mathrm{~min} 80^{\circ} \mathrm{C}, 50{ }^{\circ} \mathrm{C} /$ min ramp to $190{ }^{\circ} \mathrm{C}, 6{ }^{\circ} \mathrm{C} / \mathrm{min}$ ramp to $350{ }^{\circ} \mathrm{C}$, and $5 \mathrm{~min}$ hold at $350{ }^{\circ} \mathrm{C}$. The detector temperature was $370{ }^{\circ} \mathrm{C}$. A triglyceride standard mixture (palm kernel triglycerides, BCR 632 A, Sigma-Aldrich, St. Louis, USA) was included in each sequence to calibrate the retention times of the triglycerides. Pure cholesterol (Sigma-Aldrich) was used to calibrate the retention time of cholesterol. Triglycerides (C44, C46, $\mathrm{C} 48, \mathrm{C} 50, \mathrm{C} 52$, and C54) and cholesterol were quantified by determining the AUC. The relative triglyceride and cholesterol levels were calculated by dividing the AUCs of triglycerides and cholesterol obtained upon treatment with the test compounds by the AUCs of triglycerides and cholesterol obtained with the solvent control sample. Statistical differences were assessed by performing a one-way ANOVA followed by Dunnett's test on the normalized data (fold-change compared to the solvent control) using Graphpad Prism 5, considering a $p$ value $<0.05$ as being statistically significant. The concentration-response data were also used for BMD analysis as described below.

\section{Whole-genome gene expression analysis: microarray hybridisations and analysis}

To obtain insight into the molecular and cellular effects of PFASs in the liver, differentiated HepaRG cells were exposed for 6,24 , or $72 \mathrm{~h}$ to $100 \mu \mathrm{M}$ PFOA and for $24 \mathrm{~h}$ to $100 \mu \mathrm{M}$ PFOS or $100 \mu \mathrm{M}$ PFNA. After exposure, total RNA was isolated and purified using the RNeasy Minikit (Qiagen). RNA quality and integrity were assessed using the RNA 6000 Nano chips on the Agilent 2100 Bioanalyzer (Agilent Technologies, Amsterdam, The Netherlands). Purified RNA (100 ng) was labeled with the Ambion WT expression kit (Invitrogen) and hybridized to Affymetrix Human Gene 2.1 ST arrays (Affymetrix, Santa Clara, CA). Hybridization, washing, and scanning were carried out on an Affymetrix GeneTitan platform according to the instruction by the manufacturer. Quality control analysis, array normalization, and statistical analyses were carried out using MADMAX (Lin et al. 2011). For array normalization, the Robust Multiarray Average method (Bolstad et al. 2003; Irizarry et al. 2003) was applied. Probe sets were defined according to Dai et al. (2005). In this method, probes are assigned to Entrez IDs as a unique gene identifier. Subsequently, data were filtered with IQR 0.25 and three arrays with signal $>20 . p$ values for the effect of the PFAS treatments were calculated using an Intensity-Based Moderated T-statistic (IBMT) (Sartor et al. 2006). Significantly differentially expressed genes (DEGs) were selected using a $p$ value of $<0.001$ and foldchange of $>1.5$ (treatment versus DMSO control) as cutoffs. Hierarchical clustering of DEGs was performed using the coolmap function (R/coolmap.R) in limma. The logexpression values were prepared by subtracting from each value the mean $\log 2(\mathrm{FC})$ of the DMSO controls. Clustering of these control-corrected $\log 2(\mathrm{FC})$ expression values was performed using complete linkage and Euclidean distance. Pathway analysis on DEGs was performed using ConsensusPathDB (CPDB). CPDB analysis was applied using the webtool https://cpdb.molgen.mpg.de, which combines and compares the results of multiple pathway databases (Kamburov et al. 2011; Herwig et al. 2016). For the analysis in the present study, four databases were selected, i.e., Reactome, KEGG, Wikipathways, and Biocarta. Pathways with a $p$ value $<10^{-4}$ were considered to be affected significantly.

In a next step, MADMAX was used to perform a gene set enrichment analysis (GSEA) of the normalized and filtered data to identify enriched gene sets (Subramanian et al. 2005). Using this approach, genes are ranked based on the paired IBMT statistics and subsequently analyzed for overor underrepresentation in predefined gene sets derived from Gene Ontology, KEGG, National Cancer Institute, PFAM, Biocarta, Reactome, and WikiPathways pathway databases. Only gene sets consisting of more than 15 and fewer than 500 genes were taken into account. Statistical significance of GSEA results was determined using 1000 permutations.

\section{Real-time qPCR}

For selected genes, concentration-dependent expression levels were determined in HepaRG cells. To that end, HepaRG cells were exposed to increasing concentrations of PFOA, PFOS, or PFNA for $24 \mathrm{~h}$ and total RNA was extracted from the HepaRG cells using the RNeasy Mini Kit (Qiagen, Venlo, The Netherlands). Subsequently, 500 ng RNA was used to synthesize cDNA using the iScript cDNA synthesis kit (Bio-Rad Laboratories, Veenendaal, The Netherlands). Changes in gene expression were determined by real-time PCR on a CFX384 real-time PCR detection system (BioRad Laboratories) using SensiMix (Bioline; GC Biotech, Alphen aan den Rijn, The Netherlands). The PCR conditions 
consisted of an initial denaturation of $95^{\circ} \mathrm{C}$ for $10 \mathrm{~min}$, followed by 40 cycles of denaturation at $95{ }^{\circ} \mathrm{C}$ for $10 \mathrm{~s}$ and annealing extension at $60{ }^{\circ} \mathrm{C}$ for $15 \mathrm{~s}$. The housekeeping gene RPL27 was used for normalization. RPL27 was chosen, since this has been reported to be one of the most stable genes based on a meta-analysis of 13,629 human gene array samples to identify the most stable expressed genes (de Jonge et al. 2007). The more commonly used beta-actin and/or GAPDH vary considerably under different experimental conditions (de Jonge et al. 2007). Primer sequences were taken from the Harvard PrimerBank and ordered from Eurogentec (Liège, Belgium). Sequences of the used primers are listed in Table 1. Statistical differences were assessed by performing a one-way ANOVA followed by Dunnett's test on the normalized data (fold-change compared to the solvent control) using Graphpad Prism 5, considering a $p$ value $<0.05$ as being statistically significant. The concentration-response data were also used for benchmark dose (BMD) analysis as described below.

\section{BMD analysis of $\mathrm{qPCR}$ and cellular triglyceride data}

To obtain more insight into possible differences in potencies of the three PFASs regarding their effects on triglyceride and cholesterol levels and on their effects on the expression of selected genes, concentration-response modeling and benchmark concentration analysis were performed using the PROAST webtool (PROASTweb version 65.2, RIVM, Bilthoven, The Netherlands, https://proastweb. rivm.nl), essentially as recommended by EFSA (EFSA Scientific Committee 2017). PROAST is particularly applied for modeling of in vivo (dose-response) data, providing information on the benchmark dose (BMD). We used the PROAST software for the analysis of in vitro (concentration-response) data, thereby providing information on the benchmark concentration (BMC). Tab-delimited text files containing data on concentration, mean effect (normalized effect to the solvent control), standard deviation, and sample size (number of biological replicates) were uploaded to the PROAST webtool and analyzed as continuous (summary) data. In the tool, data are fitted to a number of mathematical models. The models showing the best (goodness of) fit, i.e., having the lowest Akaike Information Criterion (AIC) value, were used for calculation of the BMC and the corresponding two-sided $90 \%$ BMC confidence interval given by the BMCL (lower bound of the BMC confidence interval) and the BMCU (upper bound of the BMC confidence interval). The BMC, BMCL, and BMCU were determined for a benchmark response of $50 \%\left(\mathrm{BMR}_{50}\right)$ which corresponds to a $50 \%$ increase over the background response, resulting in a $\mathrm{BMC}_{50}, \mathrm{BMCL}_{50}$, and $\mathrm{BMCU}_{50}$. In PROAST, the used definitions are CES (critical effect size), CED (critical effect dose), CEDL (lower bound of the CED), CEDU (upper bound of the CED), which are identical to BMR, $\mathrm{BMC}, \mathrm{BMCL}$, and BMCU, respectively.

\section{Results}

\section{Cell viability studies}

In the first instance, the WST-1 assay was performed to get an impression on the PFAS concentrations that affect the viability of HepaRG cells. To that end, HepaRG cells in 96-well plates were exposed for 6,24 , and $72 \mathrm{~h}$ to increasing concentrations (up to $400 \mu \mathrm{M}$ ) of PFOA, PFOS, and PFNA. PFOA did not decrease cell viability upon 6 or 24 h exposure at any of the concentrations tested, but showed a clear drop in cell viability upon $72 \mathrm{~h}$ exposure at $400 \mu \mathrm{M}$ (Fig. 2). PFOS did
Table 1 Primer sequences used for qPCR

\begin{tabular}{lll}
\hline Gene name & Primer sequence & \\
\cline { 2 - 3 } & Forward & Reverse \\
\hline RPL27 & ATCGCCAAGAGATCAAAGATAA & TCTGAAGACATCCTTATTGACG \\
ANGPTL4 & CACAGCCTGCAGACACAACTC & GGAGGCCAAACTGGCTTTGC \\
$P D K 4$ & TGGAGCATTTCTCGCGCTAC & ACAGGCAATTCTTGTCGCAAA \\
$P L I N 2$ & ATGGCATCCGTTGCAGTTGAT & GATGGTCTTCACACCGTTCTC \\
$P L I N 4$ & GGCACCAAGAACACTGTCTG & TCGTACCCATGACCATAGACTT \\
$C P T 1 A$ & TCCAGTTGGCTTATCGTGGTG & CTAACGAGGGGTCGATCTTGG \\
ADH4 & AGTTCGCATTCAGATCATTGCT & CTGGCCCAATACTTTCCACAA \\
$L S S$ & GCACTGGACGGGTGATTATGG & TCTCTTCTCTGTATCCGGCTG \\
$F D P S$ & CTCCTCCCTCAGAATGAACG & CACCCTAACGATCTGGGAGA \\
$H M G C R$ & TGATTGACCTTTCCAGAGCAAG & CTAAAATTGCCATTCCACGAGC \\
$E B P$ & CTCAGCACCTAAGACTGGACA & ACGACTAAGACCCCTGTGACA \\
$I D I 1$ & TCCATTAAGCAATCCAGCCGA & CCCAGATACCATCAGACTGAGC \\
ACAT2 & CCCAGCCAATGCTTCAGGAAT & AAGCCCACGTTTATCAGCTTC \\
\hline
\end{tabular}



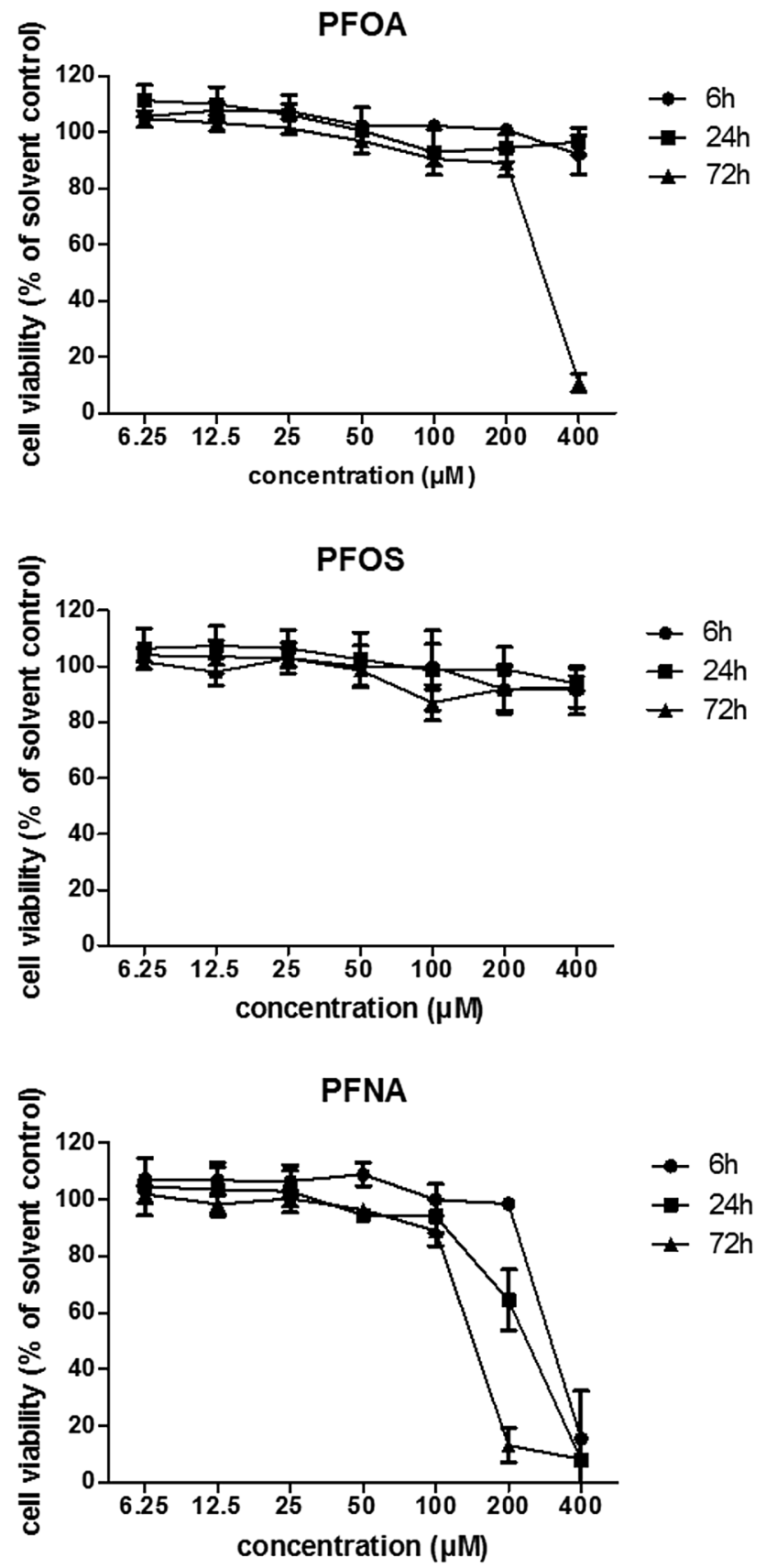

Fig. 2 Effects of 6, 24, and $72 \mathrm{~h}$ exposure to PFOA, PFOS, or PFNA on viability of HepaRG cells as determined with the WST-1 assay and expressed as \% of the solvent control (0.5\% DMSO). Data presented as mean $\pm \mathrm{SD}$ of three independent experiments (using per independent experiment the mean of three technical replicates)

not show a decrease in cell viability (not larger than 20\%) in any of the treatments $(6,24$, or $72 \mathrm{~h}$; Fig. 2). PFNA was the most cytotoxic PFAS, causing a more than $20 \%$ decrease in cell viability upon exposure for $6 \mathrm{~h}(400 \mu \mathrm{M}), 24 \mathrm{~h}(200$ and $400 \mu \mathrm{M})$ and $72 \mathrm{~h}(200$ and $400 \mu \mathrm{M})$ (Fig. 2). Cell viability upon a 24-h exposure was also determined in 24-well plates by quantifying the amount of DNA per well. These studies indicated that DNA quantity was unaffected up to $200 \mu \mathrm{M}$ PFOA, $100 \mu \mathrm{M}$ PFOA or $100 \mu \mathrm{M}$ PFNA (data not shown). Therefore, these concentrations were applied as maximum concentrations in the further studies.

\section{Triglyceride and cholesterol levels in cells}

Concentration-dependent effects of PFASs on triglyceride and cholesterol levels were determined by performing an extraction of HepaRG cells that were exposed for $24 \mathrm{~h}$ to increasing (non-cytotoxic) PFAS concentrations, followed by detection using GC-FID. The outcome of these analyses is presented in Fig. 3. All PFASs caused a concentrationdependent increase in cellular triglyceride levels. Regarding the effects of the PFASs on cholesterol levels, limited effects were noticed. A slight decrease was observed at $100 \mu \mathrm{M}$ PFOS and PFNA, but these effects were only statistically significant for PFOS. Dose-response modeling on the triglyceride data was performed using BMD analysis to obtain more insight into potency differences (Supplementary Fig. 1). $\mathrm{BMC}_{50}$ values (Table 2) indicate that PFOS and PFNA are more potent than PFOA.

\section{Microarray analysis: effects of $\mathbf{2 4} \mathrm{h}$ exposure to $100 \mu$ M PFASs}

Since none of the PFASs were cytotoxic at $100 \mu \mathrm{M}$, this concentration was used to study the effect of PFASs on wholegenome gene expression. HepaRG cells were exposed for $24 \mathrm{~h}$ to $100 \mu \mathrm{M}$ PFOA, PFOS, or PFNA, and subjected to DNA microarray analysis. For PFOA, also a time-course experiment was executed in which HepaRG cells were treated with $100 \mu \mathrm{M}$ PFOA for 6,24 , or $72 \mathrm{~h}$ followed by gene expression profiling. Figure 4 shows an overview of the results of the microarray data of HepaRG cells exposed for $24 \mathrm{~h}$ to $100 \mu \mathrm{M}$ PFOA, PFOS, or PFNA. Upon normalization and filtering of the array data, Volcano plots were generated, which show that PFNA had the largest effect on gene expression in HepaRG cells (Fig. 4a). Subsequently, differentially expressed genes (DEGs) were selected using a $p$ value of $<0.001$ (IBMT regularized paired $t$ test) and fold-change of $>1.5$ (treatment versus DMSO control) as cut-offs (for DEGs and corresponding $p$ - and fold-change values, see Supplementary Table 1). Applying these cut-offs, PFOA significantly modulated the expression of 98 genes of which 56 genes were upregulated and 42 genes were downregulated. The expression of 153 genes was found to be significantly altered by PFOS treatment ( 47 genes upregulated and 106 genes downregulated). The most pronounced effect was observed for the PFNA treatment (1024 DEGs), resulting in 476 upregulated and 548 downregulated genes (Fig. 4b). Upon hierarchical clustering of the in total 1069 DEGs, PFNA treatment is clearly separated from the other 


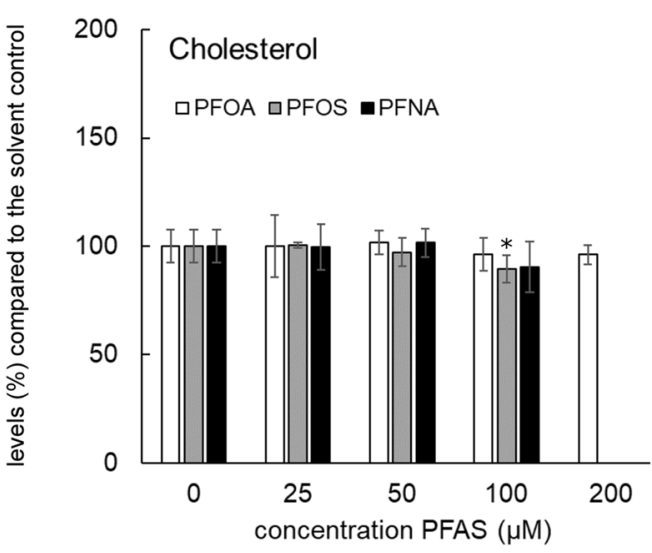

Fig. 3 Cholesterol and triglyceride levels in HepaRG cells exposed for $24 \mathrm{~h}$ to increasing concentrations of PFOA, PFOS, and PFNA. Cholesterol and triglyceride levels were normalized to the levels as measured in the solvent control, which was set at $100 \%$. Treatments

Table $2 \mathrm{BMC}_{50}$ values of PFAS-induced increase in triglycerides (sum of C44, C46, C48, C50, C52, and C54)

\begin{tabular}{lcc}
\hline & $\mathrm{BMC}_{50}(\mu \mathrm{M})$ & \\
\cline { 2 - 3 } & Expon model & Hill model \\
\hline PFOA & $184(167-201)$ & $184(167-201)$ \\
PFOS & $93(86-101)$ & $93(86-101)$ \\
PFNA & $93(86-96)$ & $92(86-96)$ \\
\hline
\end{tabular}

$\mathrm{BMC}_{50}$ values are given in $\mu \mathrm{M}$ and $\mathrm{BMCL}-\mathrm{BMCU}$ range is presented in brackets. Data are presented for the Exponential (Expon) model and the Hill model. Related concentration-response curves are presented in Supplementary Fig. 1

treatments as indicated in the heatmap, as shown in Fig. 4c. Venn diagrams of the upregulated (left) and downregulated (right) genes (Fig. 4d) show that 24 genes are commonly upregulated by PFOA, PFOS, and PFNA, and that 31 genes are commonly downregulated by these three PFASs. The expression of these genes is presented in Supplementary Fig. 2.

A concise pathway analysis was performed on these common DEGs using ConsensusPathDB (CPDB). For the 24 upregulated genes, the top 3 enriched pathway-based sets were "Amino acid synthesis and interconversion (transamination)" (Reactome), "Serine biosynthesis" (Reactome), and "PPAR signaling pathway (human)" (KEGG). For the downregulated genes, the three most significantly affected pathways were "Glycolysis/Gluconeogenesis (human)" (KEGG), "Urea cycle" (Reactome), and "Ethanol oxidation" (Reactome). More detailed results on the CPDB analysis of commonly modulated genes, as well as genes specifically regulated by one or two of the PFASs, are presented in Supplementary Table 2. Overall, the largest effects on gene

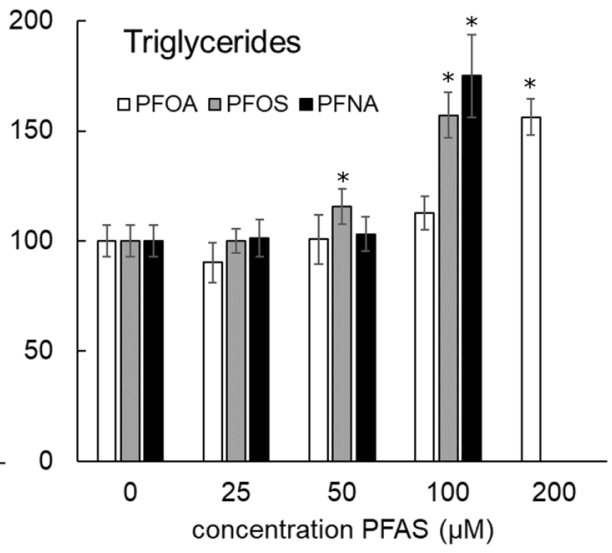

with a statistically significant difference $(p<0.05)$ in triglyceride or cholesterol levels compared to the solvent control are indicated with *. Data presented as mean \pm SD of 4 (treatments) to 12 (controls) replicates obtained in two independent experiments

expression changes were observed for PFNA, followed by PFOS and PFOA.

To obtain a better and more detailed insight into the biological pathways regulated by PFOA, PFOS, and PFNA in HepaRG cells, gene set enrichment analysis (GSEA) was performed. Figure 5 presents the top ten gene sets from these analyses for each PFAS, based on the obtained normalized enrichment scores (NERs) of significantly regulated gene sets (FDR $q$ value $<0.05$ ). Gene sets related to "PPAR signaling", "lipid metabolism", "fatty acid beta oxidation", and "tRNA amino-acylation" featured prominently among the gene sets induced by PFOA (Fig. 5a). Gene sets induced by PFOS were related to "tRNA amino-acylation", as well as to "RB (retinoblastoma protein) pathway in cancer", and "cell cycle" (Fig. 5a). For PFNA, induction of genes related to "tRNA amino-acylation", and "amino acid/oligopeptide transport" was observed (Fig. 5a). Only four gene sets were significantly repressed by PFOA, which are related to "cholesterol biosynthesis", "arginine and proline metabolism", and "glycolysis/gluconeogenesis" (Fig. 5b). "Cholesterol biosynthesis" and "glycolysis/gluconeogenesis" were also among the gene sets most prominently repressed by PFOS (Fig. 5b). PFNA repressed gene sets mainly related to "cholesterol biosynthesis" and "xenobiotics metabolism" (Fig. 5b).

The number of significantly affected gene sets by the three PFASs and the overlapping gene sets are visualized in the Venn diagram presented in Fig. 5c. When comparing all significantly regulated $(q<0.05)$ gene sets, it was found that PFOA, PFOS, and PFNA affect a number of common gene sets, including gene sets related to cholesterol biosynthesis (repressed), glycolysis/gluconeogenesis (repressed), tRNA amino-acylation (induced), amino acid transport across the cell membrane (induced), gene expression regulation by 
A

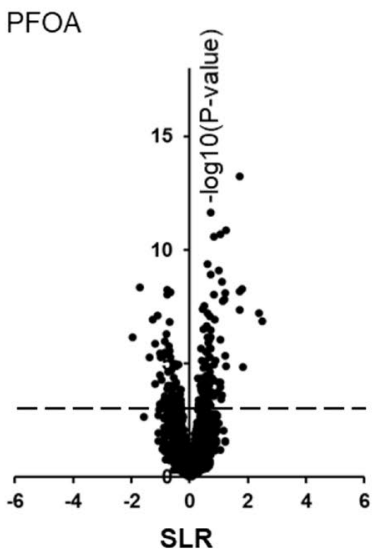

B

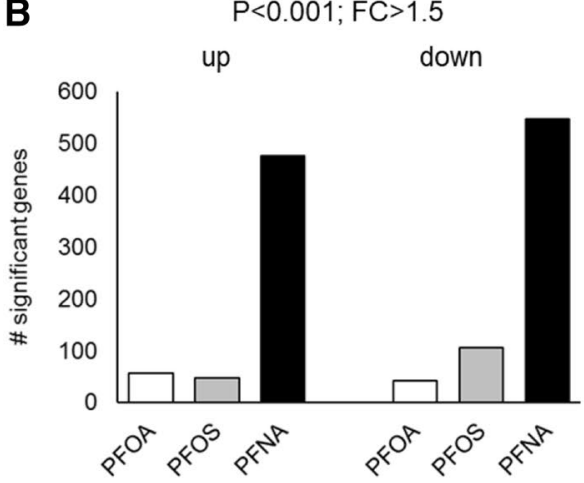

D

up

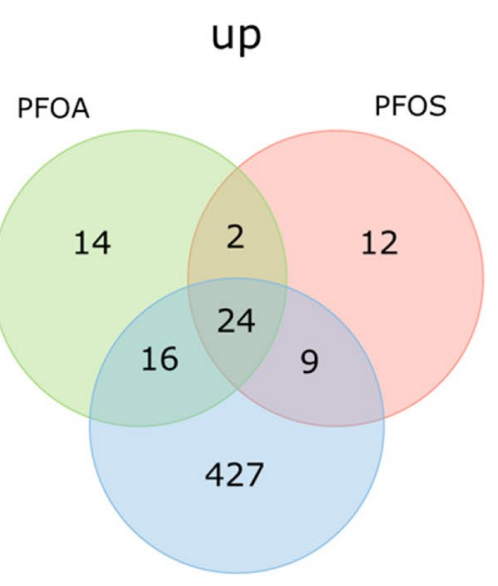

PFNA
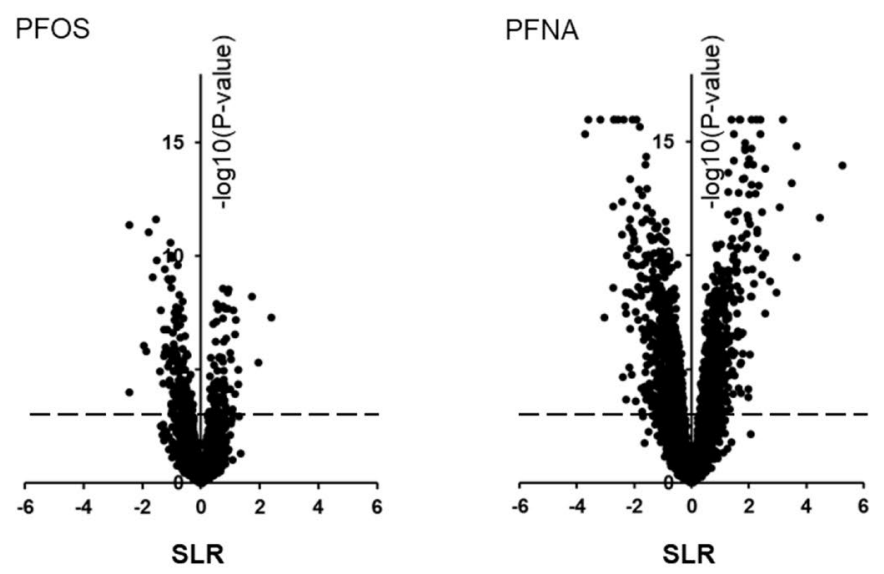

C $\quad \mathrm{P}<0.001 ; \mathrm{FC}>1.5$
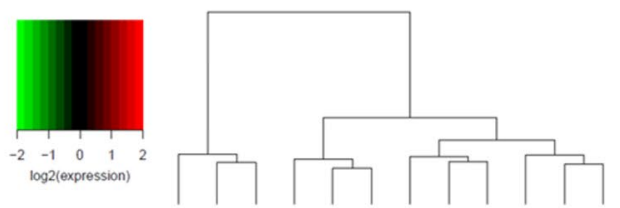

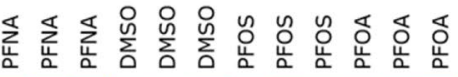

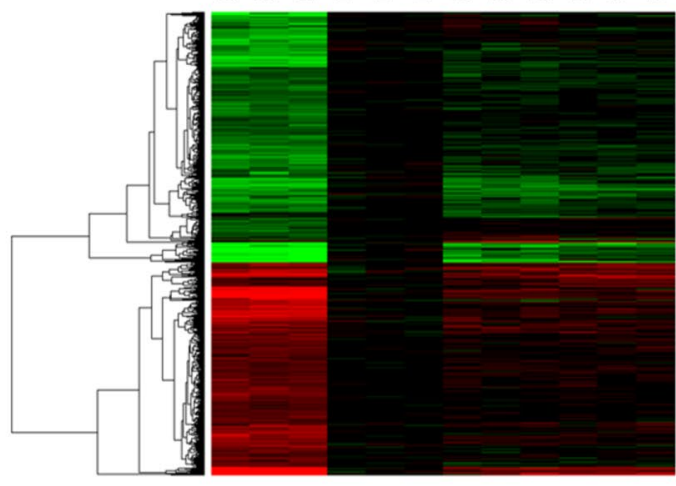

$P<0.001 ; F C>1.5$

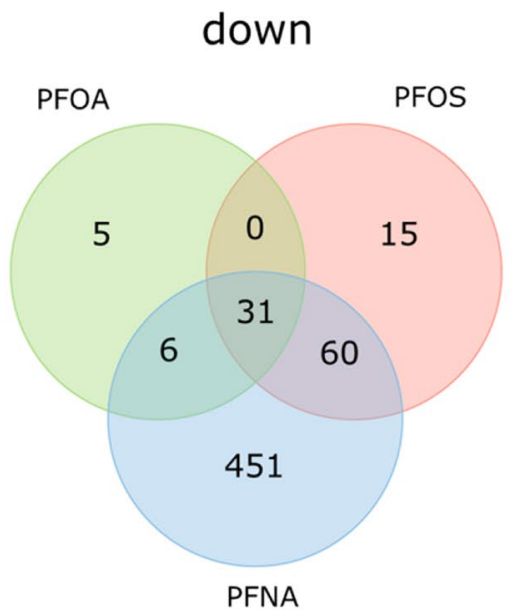


4Fig. 4 Effects of PFOA, PFOS, and PFNA on whole-genome gene expression in HepaRG cells, exposed for $24 \mathrm{~h}$ to $100 \mu \mathrm{M}$ PFOA, PFOS or PFNA. a Volcano plots showing relative changes in gene expression (expressed as signal $\log (2)$ ratio (SLR), $x$-axis) plotted against statistical significance (expressed as $-\log 10 p$ value of IBMT regularized paired $t$ test, $y$ axis). Dotted line represents cut-off of $p<0.001$. b The number of up- or downregulated genes based on a statistical significance cut-off of $p<0.001$ (IBMT regularized paired $t$ test) and a fold-change (FC) $>1.5$. c Heatmap obtained upon a hierarchical clustering of DEGs. d Venn diagrams showing the number of genes up- and downregulated by the three PFASs. Material was obtained from three independent experiments

ATF4 (induced), and gene regulation by PERK (induced; large overlap with gene sets related to gene expression regulation by ATF4).

Figure 6 shows the expression of genes related to the gene sets that were commonly repressed (Fig. 6a, b) or induced (Fig. 6c-e) by the three PFASs. As shown in Fig. 6a, the majority of the genes belonging to the gene set cholesterol biosynthesis were downregulated, showing the largest effects for PFNA, followed by PFOS, and suggesting the lowest potency for PFOA. The GSEA also indicated that the gene set related to glycolysis/gluconeogenesis was repressed (Fig. 6b). Several genes involved in glycolysis, gluconeogenesis, or both, were downregulated, whereas $P C K l$ was upregulated by PFOA and PFOS, and $P C K 2$ and $H K D C 1$ by PFNA. This gene set (KEGG_GLYCOLYSIS.GLUCONEOGENESIS) also contains several downregulated genes involved in ethanol metabolism, one of the commonly affected pathways identified with the CPDB analysis. As shown in Fig. 6c-e, the expression of genes related to ATF4related gene expression, amino acid transport across the cell membrane, and aminoacyl-tRNA biosynthesis was upregulated, showing the largest effects for PFNA, while similar effects were observed for PFOS and PFOA.

\section{Comparison of PFAS-induced gene expression with gene expression data of a typical PPARa-agonist (GW7647), LXR-agonist (GW3965), and FXR-agonist (CDCA)}

Peroxisome proliferator-activated receptor $\alpha$ (PPAR $\alpha)$ is considered to be a relevant target of PFASs and was, indeed, one of the pathways identified upon CPDB analysis and for PFOA by the GSEA (see above). To assess whether the PFASinduced expression profiles resemble the expression profile induced by a typical PPAR $\alpha$-agonist, we used the microarray data from the study of Wigger et al. (2019), who exposed HepaRG cells for 4 and $24 \mathrm{~h}$ to a series of nuclear receptor (NR) agonists [available on GEO (accession number: GSE124053)]. The data were analyzed using MADMAX as described above for the PFAS microarray data, to obtain lists of DEGs related to a 24-h exposure to GW7647 (PPAR $\alpha$ agonist), but also GW3965 [liver X receptor (LXR)-agonist] and the bile acid CDCA [farnesoid X receptor (FXR)-agonist]. Comparing these DEGs with the DEGs related to PFAS exposure indicates that the PFASs, indeed, show a gene expression profile similar to the gene expression profile induced by the PPAR $\alpha$-agonist GW7647. Typical genes include PDK4, ANGPTL4, CPTIA, and CYP4A11. This applies in particular to PFOA and PFNA, but less for PFOS (Fig. 7).

Gene expression profiles induced by the LXR-agonist GW3965 and FXR-agonist CDCA show a few similarities with the gene expression profiles induced by the PFASs (Supplementary Figs. 3, 4). Salient is that the gene most prominently downregulated by CDCA, CYP7A1, is also downregulated by all three PFASs, particularly by PFNA. This gene is known to be involved in the conversion of cholesterol to bile acids. CYP3A4 was upregulated by both the LXR-agonist GW3965 and FXR-agonist CDCA, as well as by the PFASs, and is involved in bile acid metabolism and xenobiotic metabolism.

We also assessed the effects of the three model NR agonists on the expression of genes within the gene sets commonly affected by the PFASs (Supplementary Fig. 5). These analyses indicate that the effects of model NR agonists on these genes are in general different from the effects of the PFASs.

\section{Microarray analysis: effects of 6,24 , or $72 \mathrm{~h}$ exposure to PFOA}

In addition to the analysis of a 24-h exposure to PFOA, PFOS, or PFNA, a time-course experiment was conducted in which HepaRG cells were exposed for 6, 24, or $72 \mathrm{~h}$ to $100 \mu \mathrm{M}$ PFOA, followed by DNA microarray analysis. After $6 \mathrm{~h}$ exposure, the number of significantly regulated genes was relatively low compared to $24 \mathrm{~h}$, especially for the downregulated genes (Supplementary Fig. 6). After $72 \mathrm{~h}$ of exposure, the number of significantly regulated genes was slightly higher than after $24 \mathrm{~h}$, albeit differences were small (Supplementary Fig. 6). GSEA revealed that gene sets related to "PPAR $\alpha$ signaling", "regulation of lipid metabolism by PPAR $\alpha$ ", "(mitochondrial) fatty acid beta oxidation", and "fatty acid degradation" were significantly induced at all time points (Supplementary Fig. 7). Only one and four gene sets were significantly repressed upon 6 and $24 \mathrm{~h}$ exposure, respectively, and no gene set was repressed upon a $72 \mathrm{~h}$ exposure (Supplementary Fig. 7).

\section{Gene expression analysis of selected genes related to PPAR signaling and cholesterol biosynthesis}

To gain some insight into potency differences between PFOA, PFOS, and PFNA in the PFAS-induced PPAR activation and inhibition of cholesterol biosynthesis, concentration-dependent gene expression of five selected 
Fig. 5 The top ten most strongly induced (a) or repressed (b) gene sets in HepaRG cells in response to a 24-h exposure to PFOA, PFOS or PFNA, as determined on the basis of the normalized enrichment score (NES) and statistical significance (FDR $q$ value $<0.05$ ) obtained with the gene set enrichment analysis (GSEA). For PFOA, only four gene sets were found to be repressed. c Venn diagram showing the number of gene sets induced (positive NER) and repressed (negative NER) by PFOA, PFOS, and PFNA, describing gene sets commonly affected by the three PFASs. Only gene sets were included with an FDR $q$ value $<0.05$
A

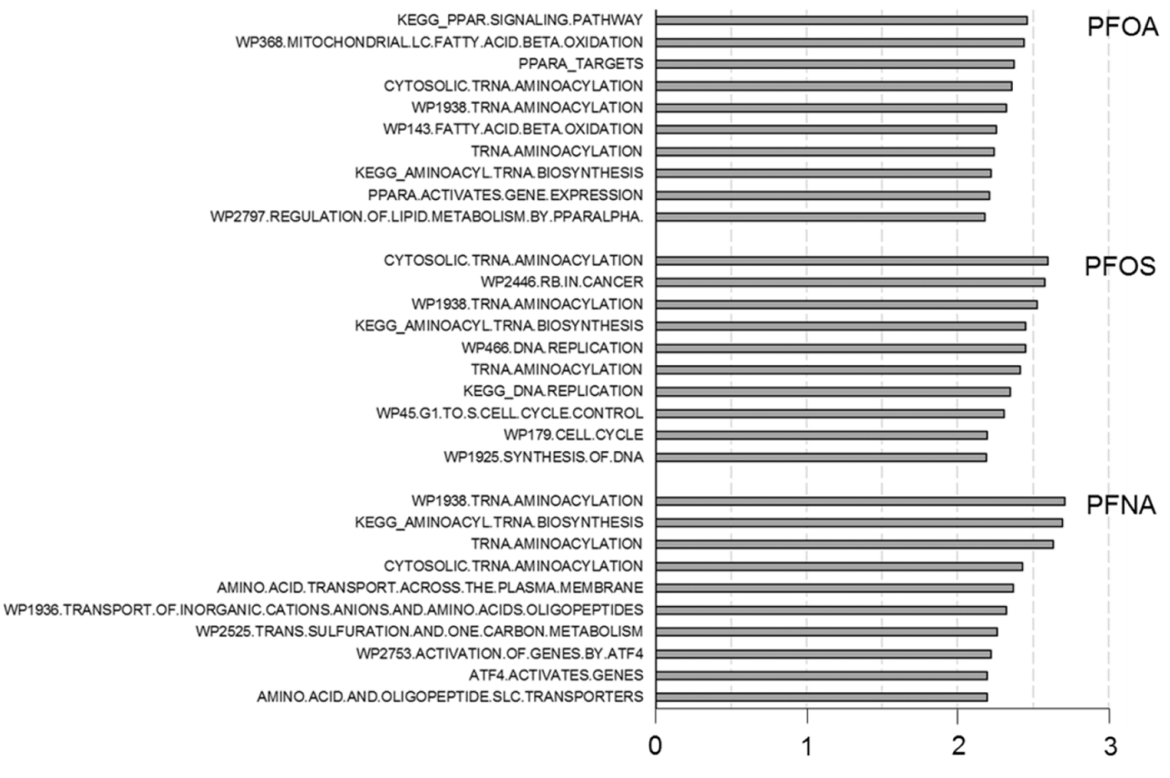

Normalized Enrichment Score

B

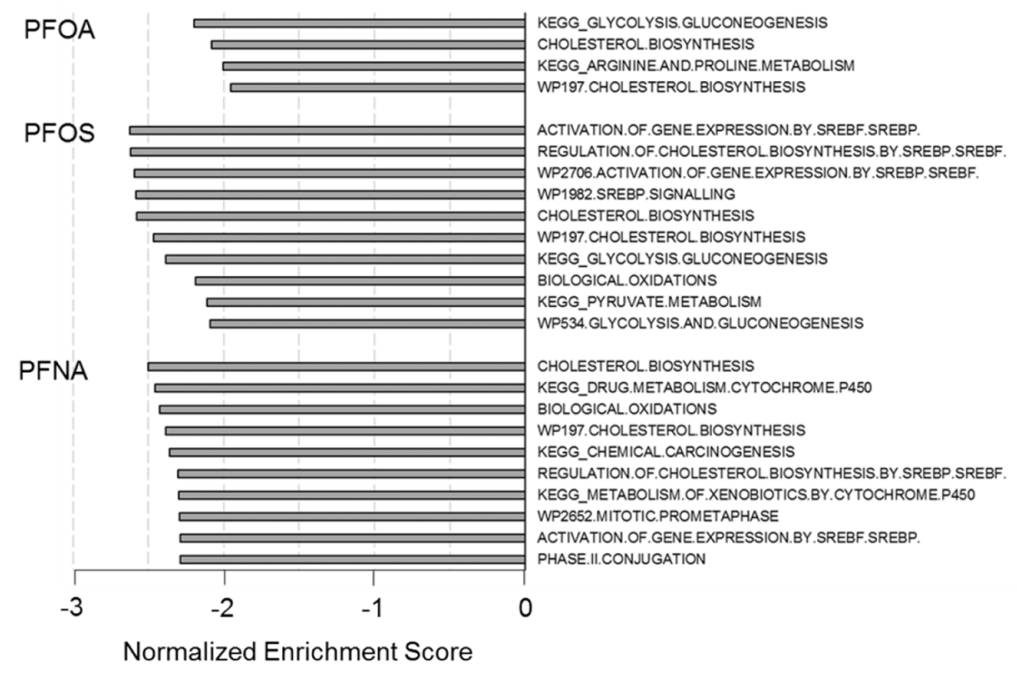

C

induced

repressed

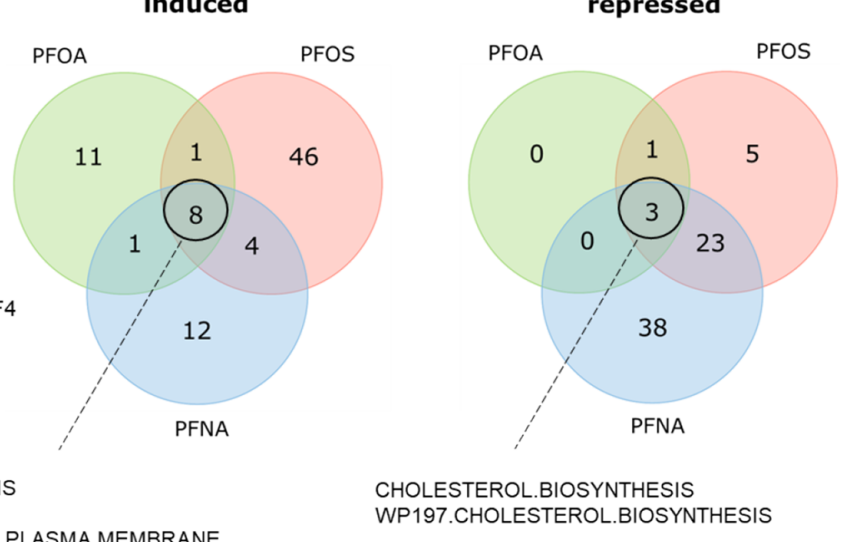

ATF4.ACTIVATES.GENES WP2753.ACTIVATION. OF.GENES.BY.ATF4

PERK.REGULATES.GENE.EXPRESSION

TRNA.AMINOACYLATION WP1938.TRNA.AMINOACYLATION CYTOSOLIC.TRNA.AMINOACYLATION KEGG_AMINOACYL.TRNA.BIOSYNTHESIS

AMINO.ACID.TRANSPORT.ACROSS.THE.PLASMA.MEMBRANE
KEGG_GLYCOLYSIS.GLUCONEOGENESIS 


\section{A}

\section{WP197.CHOLESTEROL.BIOSYNTHESIS}

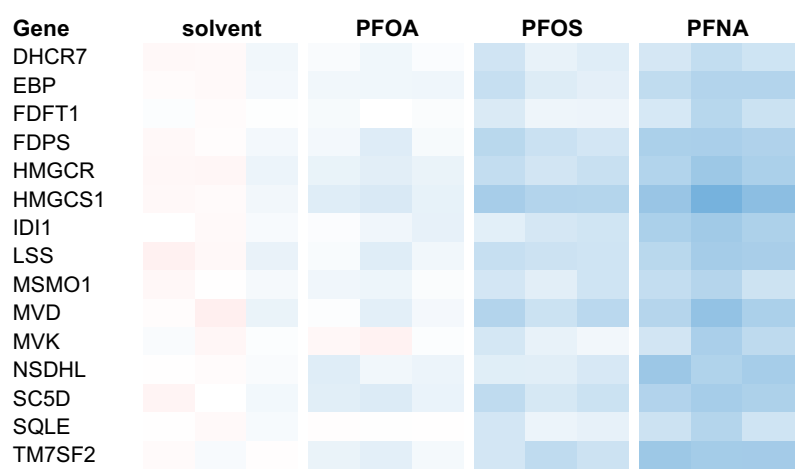

B

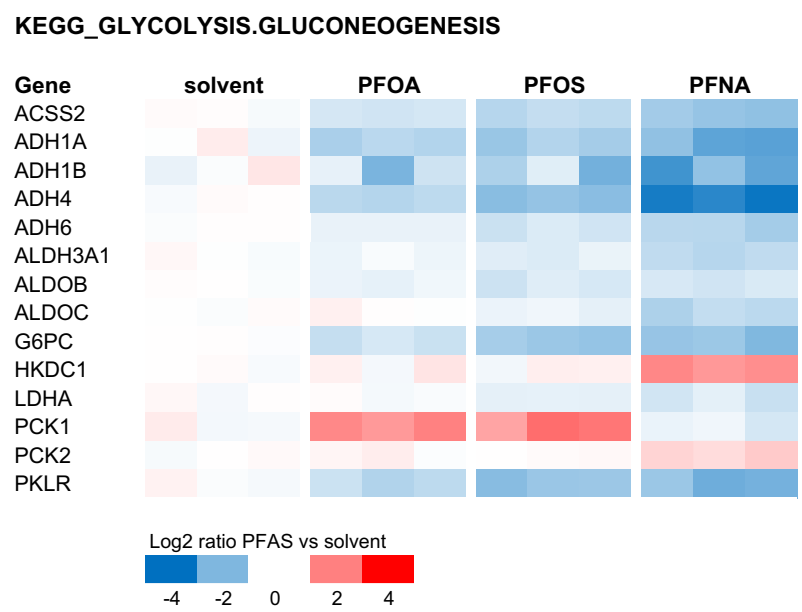

Fig. 6 PFAS-induced changes in expression of genes related to cholesterol biosynthesis (from Reactome; a), glycolysis/gluconeogenesis (from Kegg; b), PERK/ATF signaling (from Wikipathways; c), amino acid transport across the cell membrane (from Reactome; d), and aminoacyl-tRNA biosynthesis (from Kegg; e). Only genes are shown

PPAR $\alpha$ target genes (ANGPTL4, PDK4, PLIN2, PLIN4, and $C P T 1 A$ ), $A D H 4$ [the gene most downregulated by the PPAR $\alpha$ agonist GW7647, and also strongly downregulated by the PFASs (Fig. 7)] and six selected genes related to cholesterol biosynthesis (FDPS, LSS, HMGCR , EBP, IDI1, and ACAT2) was determined using qPCR analysis. Concentration-dependent increases in the expression of PLIN2, PLIN4, and PDK4 were observed for the three tested PFASs, whereas ANGPTL4 expression only significantly increased upon PFOA and PFNA exposure. Effects on $C P T 1 A$ were limited: only a small increase was noticed upon PFOA exposure. $A D H 4$ was downregulated by the three PFASs, already at relatively low concentrations, showing the highest potency for PFNA and a similar potency for PFOA and PFOS (Fig. 8).

\section{C}

\section{WP2753.ACTIVATION.OF.GENES.BY.ATF4}

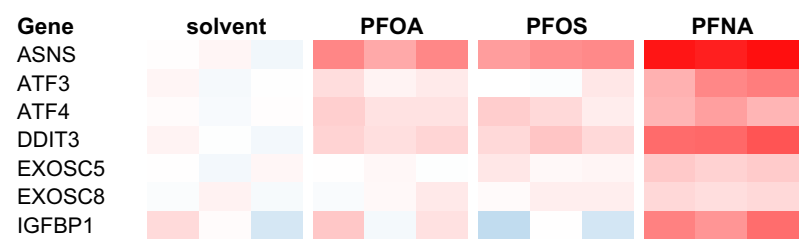

D

AMINO.ACID.TRANSPORT.ACROSS.THE.PLASMA.MEMBRANE

Gene

SLC1A4

SLC1A5

SLC38A1

SLC38A3

SLC3A2

SLC43A1

SLC7A1

SLC7A11

SLC7A2

SLC7A5

solvent
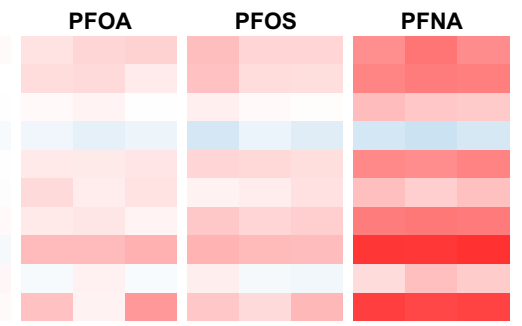

E

KEGG_AMINOACYL.TRNA.BIOSYNTHESIS

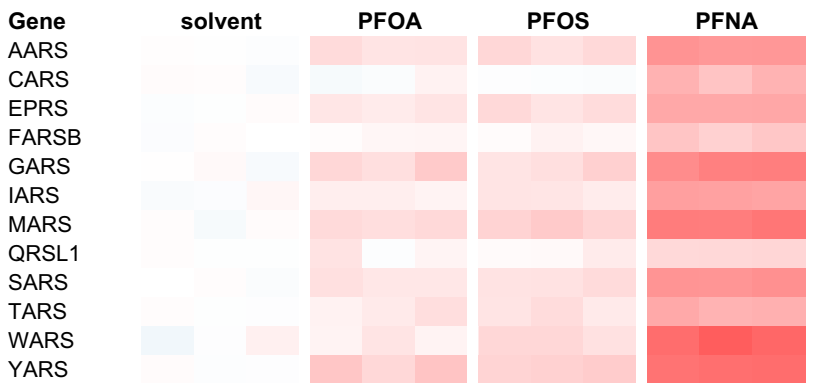

for which at least one of the PFASs induced a significant change in gene expression with at least a 1.5-fold change in expression. Genes were grouped according to the overlapping gene sets identified in the GSEA (Fig. 5c)

Concentration-dependent decreases in the expression of FDPS, LSS, HMGCR, EBP, IDII, and ACAT2 were observed for all three PFASs, although not statistically significant for IDII and ACAT2 upon PFOA exposure (Fig. 8). Concentration-response modeling using BMD analysis was performed on the normalized expression data to calculate $\mathrm{BMC}_{50}$ values (Supplementary Fig. 8). Obtained $\mathrm{BMC}_{50}$ values (Table 3) suggest that PFOA is the most potent in activating the PPAR $\alpha$ response genes followed by PFNA, although potency differences between the PFASs appeared to be gene-specific. Interestingly, PLIN2 expression was most induced by PFOS, although the BMC50 concentration was lower for PFOA. Regarding effects on the expression of cholesterogenic genes, PFNA and PFOS showed to be more potent inhibitors than PFOA (Table 3). 
Fig. 7 Gene expression changes induced by PPAR $\alpha$-agonist GW7647 (data from Wigger et al. 2019) compared with gene expression changes induced by PFASs (data from the present study) in HepaRG cells. GW7647-related DEGs for which at least one of the PFASs has a DEG are shown. Expressions of the solvent controls from the respective studies are shown left of the treatment data
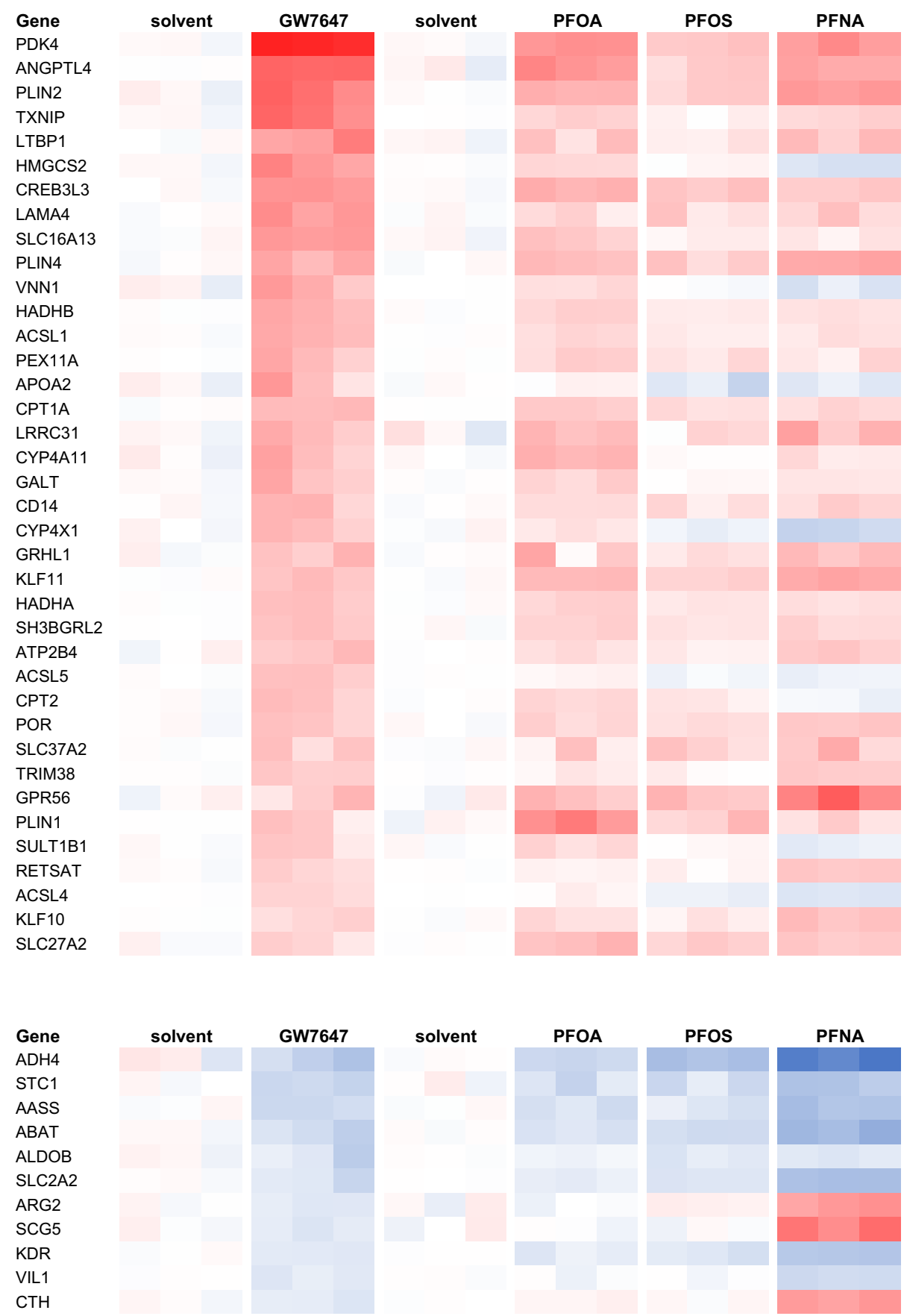

solvent
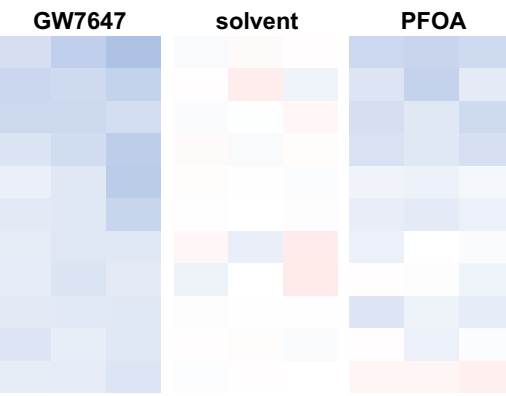

PFOS

PFNA

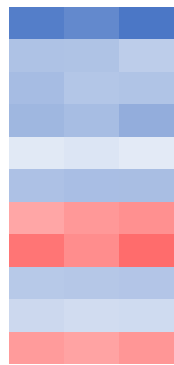

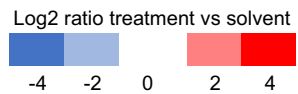

\section{Discussion}

The present study assessed the effects of PFOA, PFOS, and PFNA on human HepaRG liver cells, with a particular interest in the effects of these compounds on cholesterol and triglyceride metabolism. All three PFASs downregulated cholesterogenic genes, with PFNA being the most potent and PFOA the least potent. Despite these changes, none of the

PFASs significantly changed cellular cholesterol levels. All three PFASs caused an increase in cellular triglyceride levels, with PFOS and PFNA having similar potency and PFOA having a somewhat lower potency. Other cellular processes affected by the PFASs, as indicated by the gene expression analyses, point to effects on PERK/ATF4 signaling, tRNA amino-acylation, amino acid transport, and glycolysis/gluconeogenesis, and support the activation of PPAR $\alpha$. 
The expression of cholesterogenic genes decreased upon treatment with all three PFASs. At $100 \mu \mathrm{M}$, the strongest effects were observed for PFNA and PFOS (Fig. 6). The relatively low potency of PFOA was confirmed by concentration-response analysis of qPCR data of six selected genes of the cholesterol biosynthesis pathway, i.e., LSS, FDPS, HMGCR, EBP, IDII, and ACAT2. This qPCR analysis also suggested PFNA to be slightly more potent than PFOS in inhibiting cholesterogenic gene expression. A decrease in expression of genes related to cholesterol synthesis was also shown by Behr et al. (2020b) for PFOA and PFOS in HepaRG cells. Cellular cholesterol biosynthesis is regulated by intracellular cholesterol levels, which is mediated by sterol regulatory element-binding proteins (SREBPs) 1 and 2 (Shimano 2001; Horton et al. 2003; Adams et al. 2004; DeBose-Boyd and Ye 2018). SREBPs are synthesized as inactive precursors and held in a tripartite complex with SREBP cleavage-activating protein (SCAP) and INSIG-1 in the endoplasmic reticulum (ER) membrane. In response to low cholesterol levels, INSIG-1 dissociates and the SCAP-SREBP complex is translocated to the Golgi apparatus for proteolytic activation. The resulting active Nterminal SREBP enters the nucleus, where it binds to sterol regulatory elements in the promoter regions of target genes, including genes involved in cholesterol synthesis. In the presence of sufficient cholesterol, cholesterol accumulates in the ER membrane, causing a conformational change in SCAP resulting in binding of SCAP to INSIG-1, release of COPII, and trapping of SCAP in the ER membranes. As a result, SREBP is not transported to the Golgi and will not be activated. Besides cholesterol, unsaturated fatty acids have been reported to prevent SREBP transport from the ER to the Golgi, thereby inhibiting SREBP signaling, which may result in a decrease in cholesterol synthesis. Unsaturated fatty acids may stabilize INSIG-1 in the ER membrane, causing SCAP (and SREBP) to remain in the ER (DeBose-Boyd and Ye 2018). Interestingly, gene sets related to the regulation of cholesterol biosynthesis by SREBP (e.g., Wikipathway 1982) were downregulated, especially by PFNA and PFOS (Fig. 5b; Supplementary Fig. 9), suggesting that the PFASs inhibit SREBP-mediated gene expression in HepaRG cells. Accordingly, it can be hypothesized that PFASs may mimic the suppressive effect of unsaturated fatty acids on SREBP signaling.

Various NRs have been reported to play a role in the control of cholesterol homeostasis, such as PPARs, LXRs, and FXRs (Ory 2004; Li and Glass 2004; Li and Chiang 2009). Several studies have assessed whether PFASs bind and activate (or inhibit) gene expression regulated by different NRs (from different species), e.g., using reporter gene systems. From these studies, it is evident that various PFASs induce PPAR $\alpha$-mediated gene expression, and that activation of other NRs, including the ER $\alpha, \mathrm{ER} \beta, \mathrm{AR}, \mathrm{CAR}, \mathrm{FXR}, \mathrm{LXR} \alpha$,
LXR $\beta$, PPAR $\beta$, PPAR $\gamma$, RAR $\alpha$, and RXR $\alpha$, seems to be limited (Vanden Heuvel et al. 2006; Wolf et al. 2014; Behr et al. 2018, 2020a). Comparison of the gene expression data obtained upon HepaRG exposure to PFASs from the present study with those obtained after HepaRG exposure to a PPAR $\alpha$-agonist (GW7647), an LXR-agonist (GW3965) and an FXR-agonist (CDCA) reported by Wigger et al. (2019), indicate that the PFASs act as PPAR $\alpha$-agonists, but not as FXR nor as LXR agonists, corroborating the data obtained in the reporter gene systems. It can, therefore, be concluded that the tested PFASs in our study, indeed, activate PPAR $\alpha$ mediated gene expression, especially PFOA and PFNA, and, to a lesser extent, PFOS (Fig. 7). The relatively low activity of PFOS compared to PFOA and PFNA has also been shown by Wolf et al. (2014) using a reporter gene assay with human PPAR $\alpha$.

Various endogenous ligands have been reported for PPAR $\alpha$, including fatty acids, various eicosanoids, oxidized phospholipids, and oleoylethanolamide (Grygiel-Gorniak 2014; Schupp and Lazar 2010). Activation of PPAR $\alpha$ is well known to promote fatty acid oxidation (Dreyer et al. 1993), which is expected to lead to a reduction in intracellular triglyceride levels. Consistent with this notion, PPAR $\alpha$ deficiency in mice leads to a fatty liver (Costet et al. 1998; Kersten et al. 1999). By contrast, in the present study, all PFASs induced a concentration-dependent increase in triglyceride levels, suggesting that the triglyceride-raising effect is independent of PPAR $\alpha$. Increased liver triglyceride levels have also been observed in laboratory animals treated with PFASs, including PPAR $\alpha$ knockout mice (Das et al. 2017). However, rather than only promoting fatty acid catabolism, PPAR $\alpha$ also induces the expression of numerous genes involved in triglyceride synthesis and storage, including several lipid droplet-associated proteins of the PLIN family. Indeed, PFASs induced the expression of the PPAR $\alpha$ target genes PLIN2 and PLIN4, with strong induction by all three PFASs (Fig. 8, Table 3). Accordingly, it is conceivable that the induction of PLIN2 and/or PLIN4 mediates the observed increase in cellular triglycerides by PFASs. The notion that different PPAR $\alpha$ agonists may have different potencies towards different target genes is called the SPPARM (selective PPAR modulator) concept (Fruchart and Santos 2019) and may explain why certain PPAR $\alpha$ target genes are most strongly induced by one PFAS, whereas others are most strongly induced by another.

Treatment with PPAR $\alpha$ agonists is known to lower plasma triglyceride levels. Because of this property, a number of PPAR $\alpha$ agonists are in clinical use for the treatment of hypertriglyceridemia and atherogenic dyslipidemia. Given that PFASs and in particular PFOA and PFNA are potent PPAR $\alpha$ activators, this group of compounds may also impact on plasma triglyceride levels. Indeed, PFASs were previously shown to markedly reduce plasma triglycerides 

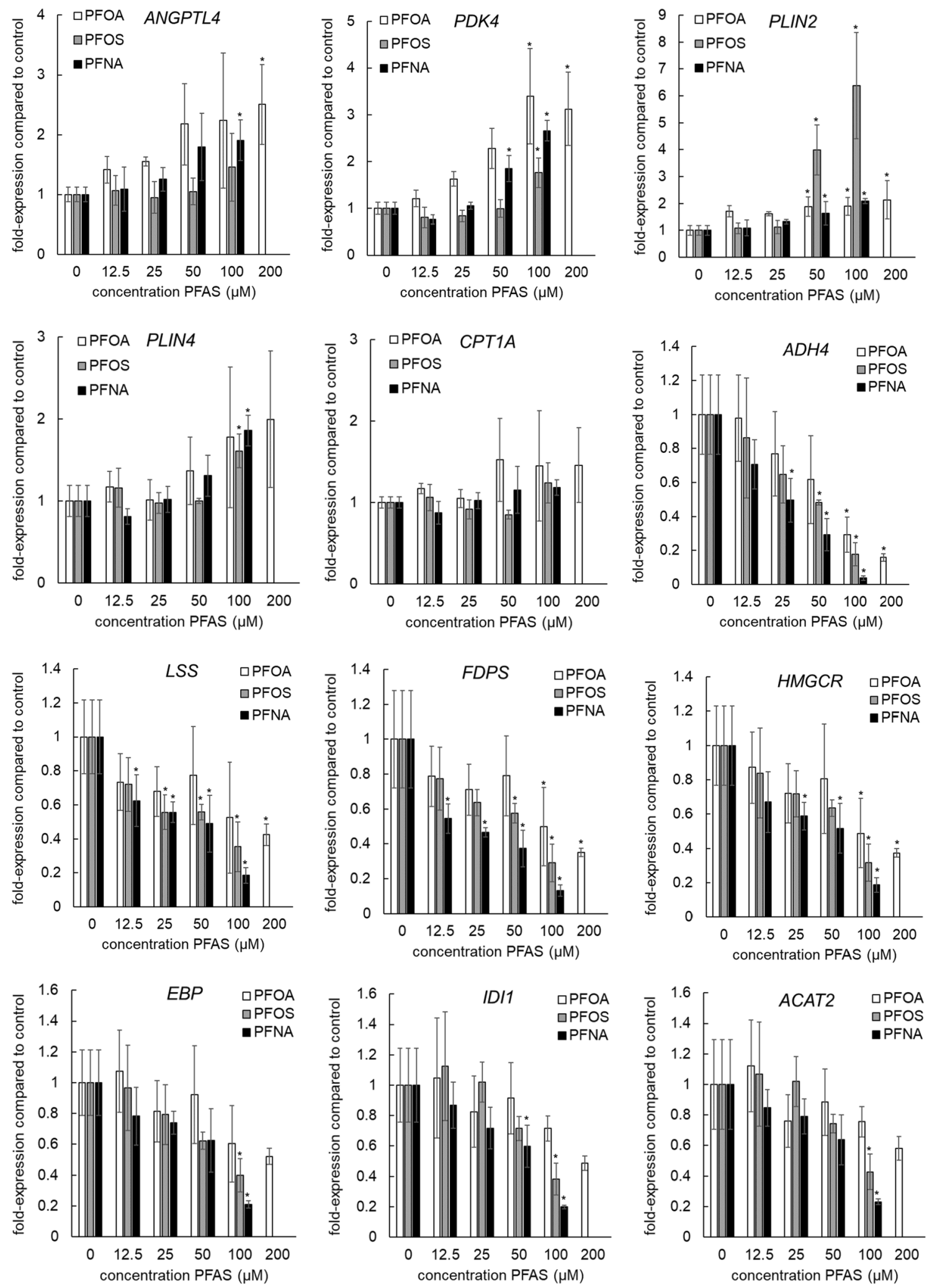

Fig. 8 Concentration-dependent effects on gene expression of the PPAR-responsive genes (ANGPTL4, PDK4, PLIN2, PLIN4, and $C P T 1 A$ ), $A D H 4$, and genes related to cholesterol biosynthesis ( $L S S$, FDPS, HMGCR, EBP, IDI1, and ACAT2) by PFOS, PFOA, and PFNA. Gene expression was normalized to the housekeeping gene RPL27 and the gene expression of the solvent control was set at 1 .
Treatments with statistically significant difference $(p<0.05)$ in gene expression compared to the solvent control are indicated with * (oneway ANOVA followed by Dunnett's multiple comparison test (Graphpad Prism 5). Data presented as mean \pm SD of three independent experiments 
in mice by stimulating lipoprotein lipase-mediated triglyceride clearance and by inhibiting the secretion of triglycerides and ApoB by the liver (Bijland et al. 2011). Intriguingly, some epidemiological studies suggest that exposure to PFASs is associated with increased rather than decreased plasma triglyceride levels (e.g., Steenland et al. 2009; Zeng et al. 2015). Whether these data reflect a causal relationship between PFASs and triglycerides remains unclear.

Epidemiological data have also revealed a positive association between plasma levels of PFASs and serum cholesterol levels (Steenland et al. 2009; Nelson et al. 2010; Eriksen et al. 2013). Currently, it is unclear whether this association reflects a causal effect of PFASs on plasma cholesterol or whether it is due to confounding. The downregulation of genes involved in cholesterol biosynthesis, also observed by Behr et al. (2020b), seems to contradict the positive association between serum cholesterol and PFASs. Nevertheless, it is interesting to speculate on potential causal mechanisms. Intracellular cholesterol levels are tightly regulated (Luo et al. 2020). Therefore, if cholesterol levels in hepatocytes would increase, these are expected to be excreted into bile (via ABCG5/8) and/or into the circulation (via VLDL particles). Hypothetically, this may result in increased serum cholesterol levels. We did not measure cholesterol levels in the medium of the cell cultures, so we cannot exclude such an effect. Behr et al. (2020b) recently measured cholesterol levels in PFOS- or PFOA-exposed HepaRG cells and the cell culture medium, using the fluorescence-based AmplexRed Cholesterol Assay. They did not find changes in cellular cholesterol levels, but reported a small increase of cholesterol in the medium upon a 24-h exposure to $100 \mu \mathrm{M}$ PFOS, but not at lower concentrations, nor by PFOA. Another hypothesis on a possible mechanism underlying increased serum cholesterol levels can be based on the data presented here, in which we find a marked repression of the SREBP pathway by PFOS and PFNA (Supplementary Fig. 9), including downregulation of the LDL receptor gene $(L D L R)$. It can be hypothesized that PFASs may be able to raise plasma cholesterol by suppressing SREBP-dependent transcription of $L D L R$, thus decreasing the hepatocellular uptake of cholesterol-rich LDL, which may result in an increase in serum cholesterol levels. Future studies should be directed towards further investigating the effect of PFASs on LDL receptor protein levels in human hepatocytes.

Although the three PFASs showed, in general, a different gene expression profile than the bile acid CDCA, similarities were also observed (Supplementary Fig. 4). CYP7Al, which codes for the key regulatory enzyme in the synthesis of bile acids from cholesterol, was found to be the gene most downregulated by CDCA in HepaRG cells (Wigger et al. 2019). CYP7Al was also downregulated by the three PFASs in our study as well as by PFOA and PFOS in the HepaRG study of Behr et al. (2020b). It is of interest to note that Behr et al. (2020b) also observed changes in cellular and extracellular bile acid composition, and that, dependent on the concentration and the chemical (PFOA or PFOS), cholic acid (CA) levels increased (GCA and TCA), whereas chenodeoxycholic acid (CDCA) levels decreased (GCDCA and TCDCA). At the gene expression level, our study shows a decrease in the expression of $C Y P 8 B 1$ and $C Y P 27 A 1$ upon PFNA treatment (Supplementary Table 1), playing a role in CA and CDCA formation, respectively. Bile acids can regulate their synthesis via a feedback mechanism by activating FXR, which results in increased expression of the gene NROB2 encoding small heterodimer partner (SHP), leading to a decrease in CYP7Al expression (Chiang 2009). However, in our study, $N R O B 2$ expression was downregulated by PFOS and PFNA (Supplementary Table 1), as also reported by Behr et al. (2020b) for PFOA and PFOS and the CYP7AI downregulation is, therefore, not expected to be mediated via FXR/SHP. Behr et al. (2020b) suggested CYP7Al downregulation to be mediated via downregulation of $H N F 4 A$ (based on Abrahamsson et al. (2005)), but HNF4A was only significantly downregulated in our study by PFNA, not fully supporting this mechanism. In agreement with the data of Behr et al. (2020b), we found, besides the downregulation of $C Y P 7 A 1$, also an upregulation of $C Y P 3 A 4$, which both have been described as key events in the AOP for cholestasis, and, thus, may contribute to the development of cholestasis (Vinken et al. 2013).

Gene sets related to glycolysis/gluconeogenesis were downregulated by all three PFASs, as indicated by the GSEA (Fig. 6b). However, PCK1, which codes for a key enzyme in gluconeogenesis (catalyzing the formation of P-enolpyruvate from oxaloacetate), was upregulated by PFOA and PFOS. PFNA induced an upregulation of $P C K 2$, which is a mitochondrial variant of $P C K 1$. PKLR, involved in the glycolysis (conversion of P-enolpyruvate to pyruvate), and G6PC, covering the last step in gluconeogenesis (conversion of glucose-6P to glucose), were downregulated by all 3 PFASs. In addition, the expression of $P D K 4$ was strongly upregulated, which may result in decreased activity of pyruvate dehydrogenase linking glycolysis with the citric acid cycle. When considering the changes in expression of these genes, the resulting cellular effects may point to the process of glyceroneogenesis (Hanson and Reshef 2003; Reshef et al. 2003; Nye et al. 2008). The upregulation of PCKl (and possibly also $P C K 2$ ) and downregulation of $P K L R$ may result in increased P-enolpyruvate levels, suggesting the process to be directed to gluconeogenesis. However, given the downregulation of the aldolases ( $A L D O B$ and $A L D O C$ ), glyceraldehyde 3-P conceivably is not directed towards fructose1,6-diphosphate but towards dihydroxyacetone-P, which can be used for triglyceride synthesis. Glyceroneogenesis was first described in white adipose tissue, but has also been reported to take place in the liver (Reshef et al. 2003) and 
is supported by the increased levels of cellular triglyceride levels in the present study.

The gene expression data from our study also point to disturbance of the endoplasmic reticulum (ER) membrane by the three PFASs, reflected by the activation of PERK/ATF4 signaling (Fig. 6c) (Liu et al. 2015; Rozpedek et al. 2016). ER stress induces Unfolded Protein Response (UPR) pathways via activation of protein kinase RNA-like endoplasmic reticulum kinase (PERK) and subsequent phosphorylation of Eukaryotic Initiation Factor 2 alpha (eIF2 $\alpha$ ). This activation of eIF $2 \alpha$ results in attenuation of global protein translation and triggers preferential translation of selected genes such as Activating Transcription Factor 4 (ATF4). ATF4 upregulates genes that play a role in cell recovery, adaptation to stress conditions, and restoration of cell homeostasis (Rozpedek et al. 2016), including genes that are involved in cell metabolism and nutrient transport. Interestingly, it has been demonstrated that during ER stress, e.g., caused by amino acid starvation, ATF4 induced the expression of aminoacyl-tRNA synthetase (ARS) genes and genes coding for amino acid transporters (Adams 2007; Shan et al. 2016; Krokowski et al. 2013; Han et al. 2013). These genes were found to be upregulated by the PFASs in the present study (Fig. 6d, e). Upon continued stress conditions, PERK/ATF4 may also activate downstream $C H O P$ (=DDIT3; Fig. 6c), which promotes apoptosis (Rozpedek et al. 2016). CHOP/DDIT3 expression was highly induced by PFNA (Fig. 6c). Activation of these pathways has also been reported to be induced by saturated fatty acids in human liver cells, possibly playing a role in the induction of non-alcoholic fatty liver disease (NAFLD) (Cao et al. 2012). Previously, it has been demonstrated that PFOA induces ER stress and UPR in HepG2 liver cells (Yan et al. 2015) and ER-stress-related autophagy in A549 lung cells (Xin et al. 2018).

It is of interest to note that the concentrations applied in the present study ( $\mu \mathrm{M}$ range) are relatively high compared to the serum concentrations that have been reported to relate to an increase in serum cholesterol in the epidemiological studies (nM range), especially considering differences in the free fraction of the PFASs in vitro (relatively high free fraction) versus in vivo (relatively low free fraction). Therefore, although the present study provides insight into mechanisms underlying PFAS toxicity, the effects as determined in the present study do not necessarily take place in humans at relevant exposure levels. Therefore, it would be of interest to test in the future the effects upon chronic exposure to PFASs at lower concentrations, as this may better resemble relevant human exposure. Interestingly, reported internal concentrations of PFOA in human cancer patients in a phase 1 dose-escalation trial were in the $\mu \mathrm{M}$ to $\mathrm{mM}$ range (Convertino et al. 2018), being in the range of concentrations tested in the present study. The reported decrease in serum cholesterol levels in these patients with increasing PFOA 
concentrations (Convertino et al. 2018), may relate to the decrease in cholesterogenic gene expression as identified in the present study.

In conclusion, the results of the present study point to an increase in cellular triglycerides upon PFAS exposure in HepaRG cells and a decrease in cholesterogenic gene expression. The latter is unlikely to be the indirect consequence of increased cholesterol levels, since our GC-FID analyses showed no effects of PFASs on cellular cholesterol levels. Rather, the effect may be due to PFAS-induced inhibition of SREBP signaling, as has been reported before for unsaturated fatty acids (Cao et al. 2012). Furthermore, although PFOA, PFNA, and, to a lesser extent, PFOS activated PPAR $\alpha$ signaling, an increase rather than a decrease in cellular triglyceride levels was observed in PFAS-exposed HepaRG cells. The mechanisms underlying this PFAS-induced increase in triglyceride levels remain to be unravelled, but may be related to induction of specific lipid droplet-associated proteins or to a possible induction of glyceroneogenesis. Interestingly, the PFASs also induced expression of genes related to ER disturbance, activating PERK-eIF2 $\alpha$-ATF4 signaling, which may explain the upregulation of aminoacyltRNA biosynthesis and amino acid transporters. Altogether, the present study provides more insight into the molecular effects of PFASs on triglyceride levels and cholesterogenic gene expression in human liver cells, but can neither confirm nor contradict a possible causality between PFAS exposure and increased serum cholesterol as identified in human epidemiological studies.

Acknowledgements This work was supported by the Dutch Ministry of Agriculture, Nature and Food Quality (project KB-23-002-021).

\section{Compliance with ethical standards}

Conflict of interest The authors declare no conflicts of interest.

Open Access This article is licensed under a Creative Commons Attribution 4.0 International License, which permits use, sharing, adaptation, distribution and reproduction in any medium or format, as long as you give appropriate credit to the original author(s) and the source, provide a link to the Creative Commons licence, and indicate if changes were made. The images or other third party material in this article are included in the article's Creative Commons licence, unless indicated otherwise in a credit line to the material. If material is not included in the article's Creative Commons licence and your intended use is not permitted by statutory regulation or exceeds the permitted use, you will need to obtain permission directly from the copyright holder. To view a copy of this licence, visit http://creativecommons.org/licenses/by/4.0/.

\section{References}

Abrahamsson A, Gustafsson U, Ellis E, Nilsson LM, Sahlin S, Björkhem I, Einarsson C (2005) Feedback regulation of bile acid synthesis in human liver: importance of HNF-4alpha for regulation of CYP7A1. Biochem Biophys Res Commun 330:395-399

Adams CM, Reitz J, De Brabander JK, Feramisco JD, Li L, Brown MS, Goldstein JL (2004) Cholesterol and 25-hydroxycholesterol inhibit activation of SREBPs by different mechanisms, both involving SCAP and Insigs. J Biol Chem 279:52772-52780

Adams CM (2007) Role of the transcription factor ATF4 in the anabolic actions of insulin and the anti-anabolic actions of glucocorticoids. J Biol Chem 282:16744-16753

ATSDR (2018) Toxicological profile for perfluoroalkyls. Draft for public comment June 2018. https://www.atsdr.cdc.gov/toxpr ofiles/tp200.pdf. Accessed 10 Nov 2018

Behr AC, Lichtenstein D, Braeuning A, Lampen A, Buhrke T (2018) Perfluoroalkylated substances (PFAS) affect neither estrogen and androgen receptor activity nor steroidogenesis in human cells in vitro. Toxicol Lett 291:51-60

Behr AC, Plinsch C, Braeuning A, Buhrke T (2020a) Activation of human nuclear receptors by perfluoroalkylated substances (PFAS). Toxicol In Vitro 62:104700

Behr AC, Kwiatkowski A, Ståhlman M, Schmidt FF, Luckert C, Braeuning A, Buhrke T (2020b) Impairment of bile acid metabolism by perfluorooctanoic acid (PFOA) and perfluorooctanesulfonic acid (PFOS) in human HepaRG hepatoma cells. Arch Toxicol (Epub ahead of print)

Bijland S, Rensen PC, Pieterman EJ, Maas AC, van der Hoorn JW, van Erk MJ, Havekes LM, Willems van Dijk K, Chang SC, Ehresman DJ, Butenhoff JL, Princen HM (2011) Perfluoroalkyl sulfonates cause alkyl chain length-dependent hepatic steatosis and hypolipidemia mainly by impairing lipoprotein production in APOE*3-Leiden CETP mice. Toxicol Sci 123:290-303

Bolstad BM, Irizarry RA, Astrand M, Speed TP (2003) A comparison of normalization methods for high density oligonucleotide array data based on variance and bias. Bioinformatics 19:185-193

Cao J, Dai DL, Yao L, Yu HH, Ning B, Zhang Q, Chen J, Cheng WH, Shen W, Yang ZX (2012) Saturated fatty acid induction of endoplasmic reticulum stress and apoptosis in human liver cells via the PERK/ATF4/CHOP signaling pathway. Mol Cell Biochem 364:115-129

Chiang JY (2009) Bile acids: regulation of synthesis. J Lipid Res 50:1955-1966

Convertino M, Church TR, Olsen GW, Liu Y, Doyle E, Elcombe CR, Barnett AL, Samuel LM, MacPherson IR, Evans TRJ (2018) Stochastic pharmacokinetic-pharmacodynamic modeling for assessing the systemic health risk of perfluorooctanoate (PFOA). Toxicol Sci 163:293-306

Costet P, Legendre C, Moré J, Edgar A, Galtier P, Pineau T (1998) Peroxisome proliferator-activated receptor alpha-isoform deficiency leads to progressive dyslipidemia with sexually dimorphic obesity and steatosis. J Biol Chem 273:29577-29585

Dai M, Wang P, Boyd AD, Kostov G, Athey B, Jones EG, Bunney WE, Myers RM, Speed TP, Akil H, Watson SJ, Meng F (2005) Evolving gene/transcript definitions significantly alter the interpretation of GeneChip data. Nucleic Acids Res 33:e175

Das KP, Wood CR, Lin MT, Starkov AA, Lau C, Wallace KB, Corton JC, Abbott BD (2017) Perfluoroalkyl acids-induced liver steatosis: effects on genes controlling lipid homeostasis. Toxicology 378:37-52

DeBose-Boyd RA, Ye J (2018) SREBPs in lipid metabolism, insulin signaling, and beyond. Trends Biochem Sci 43:358-368

de Jonge HJM, Fehrmann RSN, de Bont ESJM, Hofstra RMW, Gerbens F, Kamps WA, de Vries EGE, van der Zeev AGJ, te Meerman GJ, ter Elst A (2007) Evidence based selection of housekeeping genes. PLoS ONE 2:e898

Dreyer C, Keller H, Mahfoudi A, Laudet V, Krey G, Wahli W (1993) Positive regulation of the peroxisomal beta-oxidation pathway 
by fatty acids through activation of peroxisome proliferatoractivated receptors (PPAR). Biol Cell 77:67-76

EFSA Contam Panel (2018) Scientific opinion. Risk to human health related to the presence of perfluorooctane sulfonic acid and perfluorooctanoic acid in food. EFSA J 16(12):5194

EFSA CONTAM Panel (2020) Scientific opinion on the risk for human health related to the presence of perfluoroalkyl substances in food. In: Scientific opinion for consultation

EFSA Scientific Committee (2017) Update: guidance on the use of the benchmark dose approach in risk assessment. EFSA J 15:4658

Eriksen KT, Raaschou-Nielsen O, McLaughlin JK, Lipworth L, Tjønneland A, Overvad K, Sørensen M (2013) Association between plasma PFOA and PFOS levels and total cholesterol in a middleaged Danish population. PLoS ONE 8:e56969

Fruchart JC, Santos RD (2019) SPPARM alpha: the Lazarus effect. Curr Opin Lipidol 30:419-427

Grygiel-Górniak B (2014) Peroxisome proliferator-activated receptors and their ligands: nutritional and clinical implications-a review. Nutr J 13:17

Han J, Back SH, Hur J, Lin YH, Gildersleeve R, Shan J, Yuan CL, Krokowski D, Wang S, Hatzoglou M, Kilberg MS, Sartor MA, Kaufman RJ (2013) ER-stress-induced transcriptional regulation increases protein synthesis leading to cell death. Nat Cell Biol 15:481-490

Hanson RW, Reshef L (2003) Glyceroneogenesis revisited. Biochimie 85:1199-1205

Herwig R, Hardt C, Lienhard M, Kamburov A (2016) Analyzing and interpreting genome data at the network level with ConsensusPathDB. Nat Protoc 11:1889-1907

Horton JD, Shah NA, Warrington JA, Anderson NN, Park SW, Brown MS, Goldstein JL (2003) Combined analysis of oligonucleotide microarray data from transgenic and knockout mice identifies direct SREBP target genes. Proc Natl Acad Sci USA 100:12027-12032

Hutchins PM, Barkley RM, Murphy RC (2008) Separation of cellular nonpolar neutral lipids by normal-phase chromatography and analysis by electrospray ionization mass spectrometry. J Lipid Res 49:804-813

Irizarry RA, Bolstad BM, Collin F, Cope LM, Hobbs B, Speed TP (2003) Summaries of Affymetrix GeneChip probe level data. Nucleic Acids Res 31:e15

Kamburov A, Pentchev K, Galicka H, Wierling C, Lehrach H, Herwig R (2011) ConsensusPathDB: toward a more complete picture of cell biology. Nucleic Acids Res. 39:D712-D717

Kersten S, Seydoux J, Peters JM, Gonzalez FJ, Desvergne B, Wahli W (1999) Peroxisome proliferator-activated receptor alpha mediates the adaptive response to fasting. J Clin Investig 103:1489-1498

Krokowski D, Han J, Saikia M, Majumder M, Yuan CL, Guan BJ, Bevilacqua E, Bussolati O, Bröer S, Arvan P, Tchórzewski M, Snider MD, Puchowicz M, Croniger CM, Kimball SR, Pan T, Koromilas AE, Kaufman RJ, Hatzoglou M (2013) A self-defeating anabolic program leads to $\beta$-cell apoptosis in endoplasmic reticulum stressinduced diabetes via regulation of amino acid flux. J Biol Chem 288:17202-17213

Li T, Chiang JY (2009) Regulation of bile acid and cholesterol metabolism by PPARs. PPAR Res 2009:501739

Li AC, Glass CK (2004) PPAR- and LXR-dependent pathways controlling lipid metabolism and the development of atherosclerosis. $\mathrm{J}$ Lipid Res 45:2161-2173

Lin K, Kools H, de Groot PJ, Gavai AK, Basnet RK, Cheng F, Wu J, Wang X, Lommen A, Hooiveld GJ, Bonnema G, Visser RG, Muller MR, Leunissen JA (2011) MADMAX-Management and analysis database for multiple omics experiments. J Integr Bioinform 8:160
Liu Z, Lv Y, Zhao N, Guan G, Wang J (2015) Protein kinase R-like ER kinase and its role in endoplasmic reticulum stress-decided cell fate. Cell Death Dis 6:e1822

Luo J, Yang H, Song BL (2020) Mechanisms and regulation of cholesterol homeostasis. Nat Rev Mol Cell Biol 21(4):225-245

Nelson JW, Hatch EE, Webster TF (2010) Exposure to polyfluoroalkyl chemicals and cholesterol, body weight, and insulin resistance in the general US population. Environ Health Perspect 118:197-202

NTP (2019a) NTP technical report on the toxicity studies of perfluoroalkyl sulfonates (perfluorobutane sulfonic acid, perfluorohexane sulfonate potassium salt, and perfluorooctane sulfonic acid) administered by gavage to Sprague Dawley (Hsd:Sprague Dawley SD) rats. Research Triangle Park, NC: National Toxicology Program. Toxicity Report 96. https://ntp.niehs.nih.gov/ntp/htdoc s/st_rpts/tox096_508.pdf

NTP (2019b) NTP technical report on the toxicity studies of perfluoroalkyl carboxylates (perfluorohexanoic acid, perfluorooctanoic acid, perfluorononanoic acid, and perfluorodecanoic acid) administered by gavage to Sprague Dawley (Hsd:Sprague Dawley SD) rats. Research Triangle Park, NC: National Toxicology Program. Toxicity Report 97. https://ntp.niehs.nih.gov/ntp/htdocs/st_rpts/ tox097_508.pdf

Nye CK, Hanson RW, Kalhan SC (2008) Glyceroneogenesis is the dominant pathway for triglyceride glycerol synthesis in vivo in the rat. J Biol Chem 283:27565-27574

OECD (2018) Toward a new comprehensive global database of perand polyfluoroalkyl substances (PFASs): summary on updating the OECD 2007 list of per-and polyfluoroalkyl substances (PFASs). OECD Environment, Health and Safety Publications, Series on Risk Management, No. 39. https://www.oecd.org/ officialdocuments/publicdisplaydocumentpdf/?cote=ENV-JM$\operatorname{MONO}(2018) 7 \&$ doclanguage $=$ en

Ory DS (2004) Nuclear receptor signaling in the control of cholesterol homeostasis: have the orphans found a home? Circ Res 95:660-670

Pouwer MG, Pieterman EJ, Chang SC, Olsen GW, Caspers MPM, Verschuren L, Jukema JW, Princen HMG (2019) Dose effects of ammonium perfluorooctanoate on lipoprotein metabolism in APOE*3-Leiden.CETP mice. Toxicol Sci 168(2):519-534

Reshef L, Olswang Y, Cassuto H, Blum B, Croniger CM, Kalhan SC, Tilghman SM, Hanson RW (2003) Glyceroneogenesis and the triglyceride/fatty acid cycle. J Biol Chem 278:30413-30416

Rozpedek W, Pytel D, Mucha B, Leszczynska H, Diehl JA, Majsterek I (2016) The role of the PERK/eIF2 $\alpha / \mathrm{ATF} 4 / \mathrm{CHOP}$ signaling pathway in tumor progression during endoplasmic reticulum stress. Curr Mol Med 16:533-544

Sartor MA, Tomlinson CR, Wesselkamper SC, Sivaganesan S, Leikauf GD, Medvedovic M (2006) Intensity-based hierarchical Bayes method improves testing for differentially expressed genes in microarray experiments. BMC Bioinform 7:538

Schupp M, Lazar MA (2010) Endogenous ligands for nuclear receptors: digging deeper. J Biol Chem 285:40409-40415

Seacat AM, Thomford PJ, Hansen KJ, Olsen GW, Case MT, Butenhoff JL (2002) Subchronic toxicity studies on perfluorooctanesulfonate potassium salt in cynomolgus monkeys. Toxicol Sci 68:249-264

Shan J, Zhang F, Sharkey J, Tang TA, Örd T, Kilberg MS (2016) The C/ebp-Atf response element (CARE) location reveals two distinct Atf4-dependent, elongation-mediated mechanisms for transcriptional induction of aminoacyl-tRNA synthetase genes in response to amino acid limitation. Nucleic Acids Res 44:9719-9732

Shimano H (2001) Sterol regulatory element-binding proteins (SREBPs): transcriptional regulators of lipid synthetic genes. Prog Lipid Res 40:439-452

Steenland K, Tinker S, Frisbee S, Ducatman A, Vaccarino V (2009) Association of perfluorooctanoic acid and perfluorooctane 
sulfonate with serum lipids among adults living near a chemical plant. Am J Epidemiol 170:1268-1278

Subramanian A, Tamayo P, Mootha VK, Mukherjee S, Ebert BL, Gillette MA, Paulovich A, Pomeroy SL, Golub TR, Lander ES, Mesirov JP (2005) Gene set enrichment analysis: a knowledgebased approach for interpreting genome-wide expression profiles. Proc Natl Acad Sci USA 102:15545-15550

Vanden Heuvel JP, Thompson JT, Frame SR, Gillies PJ (2006) Differential activation of nuclear receptors by perfluorinated fatty acid analogs and natural fatty acids: a comparison of human, mouse, and rat peroxisome proliferator-activated receptor-alpha, -beta, and -gamma, liver $\times$ receptor-beta, and retinoid $\times$ receptor-alpha. Toxicol Sci 92:476-489

Vinken M, Landesmann B, Goumenou M, Vinken S, Shah I, Jaeschke H, Willett C, Whelan M, Rogiers V (2013) Development of an adverse outcome pathway from drug-mediated bile salt export pump inhibition to cholestatic liver injury. Toxicol Sci 136:97-106

Wang Z, DeWitt JC, Higgins CP, Cousins IT (2017) A never-ending story of per- and polyfluoroalkyl substances (PFASs)? Environ Sci Technol 51:2508-2518

Wigger L, Casals-Casas C, Baruchet M, Trang KB, Pradervand A, Naldi A, Desvergne B (2019) System analysis of cross-talk between nuclear receptors reveals an opposite regulation of the cell cycle by LXR and FXR in human HepaRG liver cells. PLoS ONE 14:e0220894

Wolf CJ, Rider CV, Lau C, Abbott BD (2014) Evaluating the additivity of perfluoroalkyl acids in binary combinations on peroxisome proliferator-activated receptor- $\alpha$ activation. Toxicology 316:43-54

Xin Y, Wan B, Yang Y, Cui XJ, Xie YC, Guo LH (2018) Perfluoroalkyl acid exposure induces protective mitochondrial and endoplasmic reticulum autophagy in lung cells. Arch Toxicol 92:3131-3147

Yan S, Zhang H, Wang J, Zheng F, Dai J (2015) Perfluorooctanoic acid exposure induces endoplasmic reticulum stress in the liver and its effects are ameliorated by 4-phenylbutyrate. Free Radic Biol Med 87:300-311

Zeng XW, Qian Z, Emo B, Vaughn M, Bao J, Qin XD, Zhu Y, Li J, Lee YL, Dong GH (2015) Association of polyfluoroalkyl chemical exposure with serum lipids in children. Sci Total Environ 512-513:364-370

Publisher's Note Springer Nature remains neutral with regard to jurisdictional claims in published maps and institutional affiliations. 